\title{
The SDSS-HET Survey of Kepler Eclipsing Binaries. Description of the Survey and First Results
}

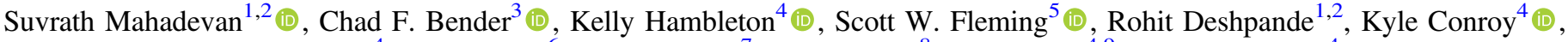 \\ Gal Matijevič ${ }^{4}$, Leslie Hebb ${ }^{6}$ (1) , Arpita Roy ${ }^{7}$ (1) , Hasan $\mathrm{Ak}^{8}$, Blaž Leban ${ }^{4,9}$, and Andrej Prša ${ }^{4}$ (D) \\ ${ }^{1}$ Department of Astronomy \& Astrophysics, The Pennsylvania State University, 525 Davey Lab, University Park, PA 16802, USA; suvrath@astro.psu.edu \\ ${ }^{2}$ Center for Exoplanets \& Habitable Worlds, The Pennsylvania State University, 525 Davey Lab, University Park, PA 16802, USA \\ ${ }^{3}$ Department of Astronomy and Steward Observatory, University of Arizona, Tucson, AZ 85721, USA \\ ${ }^{4}$ Department of Astronomy \& Astrophysics, Villanova University, 800 East Lancaster Ave., Villanova, PA 18085, USA; aprsa@ villanova.edu \\ ${ }^{5}$ Space Telescope Science Institute, 3700 San Martin Dr, Baltimore, MD 21218, USA \\ ${ }^{6}$ Physics Department, Hobart and William Smith Colleges, 300 Pulteney Street, Geneva, NY 14456, USA \\ ${ }^{7}$ California Institute of Technology, 1200 E California Blvd, Pasadena, CA 91125, USA \\ ${ }^{8}$ Erciyes University, Science Faculty, Astronomy and Space Sci. Dept., 38039 Kayseri, Turkey \\ ${ }^{9}$ University of Ljubljana, Dept. of Physics, Jadranska 19, SI-1000 Ljubljana, Slovenia \\ Received 2018 November 9; revised 2019 July 30; accepted 2019 July 30; published 2019 October 17
}

\begin{abstract}
The Kepler mission has provided a treasure trove of eclipsing binaries (EBs), observed at extremely high photometric precision, nearly continuously for several years. We are carrying out a survey of $\sim 100$ of these EBs to derive dynamical masses and radii with precisions of $3 \%$ or better. We use multiplexed near-infrared $H$-band spectroscopy from the Sloan Digital Sky Survey-III and -IV APOGEE instrument and optical spectroscopy from the Hobby-Eberly Telescope High-resolution Spectrograph to derive double-lined spectroscopic orbits and dynamical mass ratios $(q)$ for the EB sample, two of which we showcase in this paper. This orbital information is combined with Kepler photometry to derive orbital inclination, dynamical masses of the system components, radii, and temperatures. These measurements are directly applicable for benchmarking stellar models that are integrating the next generation of improvements, such as the magnetic suppression of convection efficiency, updated opacity tables, and fine-tuned equations of state. We selected our EB sample to include systems with low-mass $\left(M \lesssim 0.8 M_{\odot}\right.$ ) primary or secondary components, as well as many EBs expected to populate the relatively sparse parameter space below $\sim 0.5 M_{\odot}$. In this paper, we describe our EB sample and the analytical techniques we are utilizing, and also present masses and radii for two systems that inhabit particularly underpopulated regions of mass-radius-period space: KIC 2445134 and KIC 3003991. Our joint spectroscopic and photometric analysis of KIC $2445134(q=0.411 \pm 0.001)$ yields masses and radii of $M_{A}=1.29 \pm 0.03 M_{\odot}, M_{B}=0.53 \pm 0.01 M_{\odot}$, $R_{A}=1.42 \pm 0.01 R_{\odot}, R_{B}=0.510 \pm 0.004 R_{\odot}$, and a temperature ratio of $T_{B} / T_{A}=0.635 \pm 0.001$; our analysis of KIC $3003991 \quad(q=0.298 \pm 0.006)$ yields $M_{A}=0.74 \pm 0.04 M_{\odot}, \quad M_{B}=0.222 \pm 0.007 M_{\odot}$, $R_{A}=0.84 \pm 0.01 R_{\odot}, R_{B}=0.250 \pm 0.004 R_{\odot}$, and a temperature ratio of $T_{B} / T_{A}=0.662 \pm 0.001$.
\end{abstract}

Key words: binaries: eclipsing - stars: fundamental parameters - stars: low-mass - techniques: photometric techniques: radial velocities - techniques: spectroscopic

\section{Introduction}

Eclipsing binaries (EBs) have served as benchmarks for stellar astrophysics for hundreds of years (e.g., Goodricke 1783). Advances in the precision of radial velocity (RV) measurements over the past several decades have steadily improved the precision of masses derived from EBs. Compilations of EBs show that the total number, diversity, and measurement precisions of detached, main-sequence EBs have steadily increased: 72 stars with masses and radii $(M, R)$ measured to $<15 \%$ in Popper (1980), 88 stars with $M, R<2 \%$ in Andersen (1991), 188 stars with $M, R<3 \%$ in Torres et al. (2010), and 198 stars with $M, R<2 \%$ in DEBCat, ${ }^{10}$ an updated version of Andersen (1991).

Despite this steady growth in sample size, low-mass stars (defined here as $M<0.8 M_{\odot}$ ) remain a relatively small fraction of the overall sample. For example, DEBCat contains only 54 stars with $M<0.8 M_{\odot}$, while the sample of Torres et al. (2010) has only 10 such stars, none of which has an orbital period longer than five days. More recent results are starting to expand

\footnotetext{
$\overline{10}$ http://www.astro.keele.ac.uk/jkt/debcat/
}

the low-mass sample (e.g., Schwamb et al. 2013; Gómez Maqueo Chew et al. 2014; Zhou et al. 2015; Dittmann et al. 2017; Lubin et al. 2017; Casewell et al. 2018), but a significant increase in sample size is still lacking, predominantly due to the resource expense associated with obtaining high-precision RVs (the method used to derive most of the EB dynamical masses in the current compilations), and because the poor flux ratio of a low-mass $\mathrm{K} / \mathrm{M}$ dwarf orbiting a larger primary makes it difficult to detect in the optical.

Observations in the near-infrared (NIR) result in the tangible benefit of improving the flux contrast for EBs with a small mass ratio $\left(q=M_{B} / M_{A}\right)$. For example, the flux ratio of an M5 dwarf, with $T_{\text {eff }} \sim 3300 \mathrm{~K}$, to a G2 dwarf, with $T_{\text {eff }} \sim 5800 \mathrm{~K}$, is $\sim 10$ times more favorable in the $H$ band than in the $V$ band. This allows for the extraction of measured RVs from fainter secondary stars, and therefore extends the lower limit on $q$ for which masses of dwarf stars can be derived. While optical spectra tend to lose sensitivity at $q \sim 0.5$, NIR spectra can push down to $q \sim 0.1$ (Prato et al. 2002; Mazeh et al. 2003; Bender $\&$ Simon 2008).

Measurements of selected bright binaries have achieved precisions in mass ratio (in $M \sin i$ ) of $0.02 \%-0.42 \%$ (Konacki 
et al. 2010), but precisions of 1\%-3\% are still sufficient to distinguish between model parameters (Morales et al. 2010). The high photometric precision of Kepler makes it possible to determine the masses of some EBs purely photometrically (Carter et al. 2011; Faigler et al. 2012) via Doppler boosting and ellipsoidal effects (Faigler \& Mazeh 2011). While the technique is promising, Carter et al. (2011) achieved only a $10 \%$ mass precision using the Kepler photometry alone (i.e., without including any spectroscopic RVs), while Faigler et al. (2012) found that only five of their seven binaries had RV semi-amplitudes in agreement with those predicted from their photometric analysis. In a large follow-up program, Tal-Or et al. (2015) observed 281 targets, confirming 70 binary systems while finding that many of their false positives were due to pulsating red giants. The use of spectroscopic RVs therefore remains an essential and reliable method for obtaining mass precisions at the $1 \%-3 \%$ level, motivating the continual use of spectra to measure the stellar masses of EBs.

Stellar models rely on the precision measurements that EBs afford for calibrating the physical parameters used in their calculations (leading to well characterized EBs often being referred to as stellar "benchmarks"). Stars with $M>0.8 M_{\odot}$ generally agree with theoretical models to within observational uncertainties. Lower-mass stars, however, are often observed to have radii that are 5\%-15\% larger than model predictions (López-Morales 2007; Morales et al. 2008; Torres et al. 2010; Kraus et al. 2011; Higl \& Weiss 2017; Cruz et al. 2018; Kesseli et al. 2018). There is observational and theoretical evidence to suggest that magnetic fields could be the cause (Chabrier et al. 2007; Morales et al. 2010; Feiden \& Chaboyer 2013; MacDonald \& Mullan 2017), due to interaction with the partially convective outer atmospheres and/or generation of cool starspots at polar latitudes. Unidentified opacity sources have also been suggested (Berger et al. 2006), although a lack of metallicity measurements for many stars in the current EB sample has prevented an in-depth examination of that possible correlation. Models that use a different equation of state than previous works agree with observations of one low-mass Kepler EB (KOI-126; Feiden et al. 2011), opening another regime of parameter space to explore.

In this paper we introduce the SDSS-HET Survey of Kepler $E B s$, which is combining Kepler photometry with groundbased spectroscopy to precisely measure orbital parameters, dynamical masses and radii, and temperature ratios for a sample of 109 EB candidates selected from the catalog of Kirk et al. (2016) and listed in Table 1. We utilize both optical and NIR spectroscopy to solve these EBs as double-lined spectroscopic binaries (SB2s), and combine these results with Kepler photometry to derive masses and radii with precisions of better than $3 \%$ for most of the sample, and as good as $1 \%$ for a subset. Here we present two objects in our sample. Our complete sample is restricted to EBs that are classified as fully detached and have $H<13$. Orbital periods range from a few days to more than 100 days. The total sample size is comparable in number to the $M<2 M_{\odot}$ members in Torres et al. (2010) and DEBCat (110 and $285 \mathrm{EBs}$, respectively), but we have included many EBs with low-mass primary or secondary components in order to substantially increase the population of well measured low-mass stars. One of the reasons why the compilation from Torres et al. (2010) is so useful to the astronomical community is that their study homogeneously recomputed orbital and stellar parameters from the compiled list of literature EBs. Our survey will intrinsically possess this quality, because our analysis applies a singular set of tools to a homogeneous data set.

In Section 2 we describe the facilities and data products we are using, and in Section 3 we discuss the analytical techniques applied to the spectroscopy and photometry, as well as probabilistic analysis that we use to derive realistic parameter uncertainties. In Section 4 we present two low-mass EBs from our sample, and provide our derived orbits, masses, and radii for these systems. In Section 5 we discuss these systems in the context of the pre-existing population of precisely measured low-mass EBs, and describe our plans for analyzing the remainder of our Kepler EB sample. As part of this program, we constructed a semi-automated reduction pipeline for the HET High-resolution Spectrograph, which we describe in the Appendix.

\section{Facilities and Data Sets}

\subsection{The Kepler Mission}

Kepler is a space-borne, $0.95 \mathrm{~m}$, high-precision photometer equipped with a broadband filter covering 420-865 nm. From 2009 to 2013, Kepler monitored a single field located 13.5 above the Galactic plane in the direction of Cygnus, with a mission to detect transiting, habitable-zone exoplanets (Borucki et al. 2010). Its ability to conduct photometry with high precision $\left(\sim 80\right.$ ppm for $K_{p}=12$; Caldwell et al. 2010$)$ and to observe with a nearly continuous cadence facilitated numerous ancillary stellar astrophysics programs, including the detection of EBs over a range of orbital periods that are challenging to observe from the ground. The majority of Kepler EBs are faint (only $\sim 12 \%$ of detached systems have $K_{p}<12$ in Kirk et al. 2016), and follow-up spectroscopy of such systems requires a combination of large telescopes and long exposure times. The Kepler EB Catalog ${ }^{11}$ (Prša et al. 2011; Slawson et al. 2011; Matijevič et al. 2012; Conroy et al. 2014; Abdul-Masih et al. 2016; Kirk et al. 2016) has used Kepler photometry to identify thousands of EBs, and has derived extremely precise orbital periods, which greatly simplifies the process of turning individual spectroscopic measurements into an SB2 orbit.

\subsection{The SDSS-III APOGEE Spectrometer}

The Apache Point Observatory Galactic Evolution Experiment (APOGEE, Majewski et al. 2017) is a fiber-fed, multiobject, near-infrared spectrometer that uses a volume phase holographic grating and a linear array of three Hawaii-2RG detectors to record spectra from 1.51 to $1.68 \mu \mathrm{m}$ with a spectral resolution of $\lambda / \Delta \lambda \sim 22,500$ (Wilson et al. 2010). The instrument is located at Apache Point Observatory on the $2.5 \mathrm{~m}$ telescope of the Sloan Digital Sky Survey (SDSS) (York et al. 2000; Gunn et al. 2006), and was commissioned in the spring and summer of 2011 for a three-year survey aimed at a Galactic evolution experiment (Allende Prieto et al. 2008; Eisenstein et al. 2011). A small fraction of the survey time $(\sim 5 \%)$ was devoted to ancillary science programs (Zasowski et al. 2013), which include the Kepler EB program described in this paper. The spectrograph is stabilized in a vacuum-sealed cryostat cooled via liquid nitrogen, which minimizes thermal variations and yields a typical RV precision of 100-200 $\mathrm{m} \mathrm{s}^{-1}$ on our EB sample. APOGEE can simultaneously observe 300

\footnotetext{
${ }^{11} \mathrm{http}: / /$ keplerebs.villanova.edu/
} 
Table 1

Project EBs Observed

\begin{tabular}{|c|c|c|c|c|c|c|c|}
\hline KIC ID & $K_{p}$ & $g$ & $H$ & $P_{\text {cat }}$ & $N_{\mathrm{APG}}$ & $N_{\mathrm{HET}}$ & Notes \\
\hline 01571511 & 13.424 & 13.855 & 12.041 & 14.022451 & 3 & 9 & \\
\hline 02010607 & 11.347 & 11.630 & 10.204 & 18.632296 & 3 & 6 & \\
\hline 02162994 & 14.162 & 14.696 & 12.570 & 4.101595 & 3 & 6 & \\
\hline 02305372 & 13.821 & 14.341 & 12.201 & 1.404691 & 3 & 0 & \\
\hline 02305543 & 12.545 & 12.971 & 11.138 & 1.362274 & 3 & 0 & \\
\hline 02306740 & 13.545 & 14.025 & 12.022 & 10.306987 & 3 & 7 & \\
\hline 02308957 & 14.520 & 15.031 & 12.916 & 2.219684 & 3 & 6 & \\
\hline 02309587 & 13.925 & 14.434 & 12.359 & 1.838511 & 3 & 0 & \\
\hline 02309719 & 12.899 & 13.335 & 11.528 & 54.356360 & 3 & 9 & \\
\hline 02445134 & 13.551 & 13.948 & 12.201 & 8.412009 & 3 & 8 & \\
\hline 02445975 & 13.513 & 14.404 & 12.830 & 6.777765 & 3 & 0 & \\
\hline 02447893 & 14.490 & 15.134 & 12.628 & 0.661620 & 3 & 0 & \\
\hline 02576692 & 12.744 & 13.191 & 11.371 & 87.878533 & 3 & 8 & \\
\hline 02583777 & 12.735 & 13.166 & 11.311 & 0.958117 & 3 & 0 & \\
\hline 02708156 & 10.672 & 10.672 & 10.525 & 1.891272 & 3 & 0 & \\
\hline 02711114 & 12.335 & 12.634 & 11.115 & 2.858880 & 3 & 0 & \\
\hline 02720354 & 13.116 & 13.387 & 11.965 & 2.821328 & 3 & 9 & \\
\hline 02860594 & 13.370 & 13.613 & 12.237 & 5.499945 & 3 & 10 & \\
\hline 02860788 & 14.043 & 14.614 & 12.255 & 5.259742 & 3 & 0 & \\
\hline 02997455 & 13.800 & 14.694 & 11.500 & 1.129850 & 3 & 0 & EB Cat FP, No RV Var \\
\hline 03003991 & 13.926 & 14.482 & 12.278 & 7.244779 & 6 & 8 & \\
\hline 03120320 & 10.885 & 11.280 & 9.611 & 10.265613 & 3 & 6 & \\
\hline 03127817 & 12.155 & 12.242 & 10.590 & 4.327139 & 6 & 9 & \\
\hline 03128793 & 14.633 & 15.546 & 12.310 & 24.679381 & 6 & 0 & \\
\hline 03130300 & 14.313 & 14.696 & 12.970 & 11.531282 & 6 & 0 & EB Cat FP, No RV Var \\
\hline 03230578 & 13.406 & 13.860 & 12.189 & 6.337611 & 3 & 0 & \\
\hline 03230787 & 12.553 & 12.990 & 11.141 & 17.734052 & 3 & 8 & \\
\hline 03241619 & 12.524 & 13.063 & 10.798 & 1.703344 & 3 & 0 & \\
\hline 03247294 & 13.924 & 14.353 & 12.442 & 67.418828 & 6 & 7 & \\
\hline 03248033 & 12.161 & 12.427 & 11.019 & 2.668220 & 6 & 0 & EB Cat FP, No RV Var \\
\hline 03248332 & 13.102 & 13.369 & 12.012 & 7.363607 & 6 & 6 & \\
\hline 03335816 & 12.084 & 12.399 & 10.842 & 7.422006 & 3 & 7 & \\
\hline 03339538 & 13.391 & 14.106 & 11.429 & 14.658014 & 3 & 5 & \\
\hline 03351945 & 14.734 & 15.549 & 12.605 & 1.080538 & 6 & 0 & \\
\hline 03352751 & 13.444 & 13.541 & 12.304 & 3.495455 & 6 & 9 & \\
\hline 03439031 & 11.287 & 11.503 & 10.117 & 5.952026 & 3 & 6 & \\
\hline 03440230 & 13.636 & 13.706 & 12.486 & 2.881101 & 3 & 0 & \\
\hline 03441784 & 9.729 & 9.898 & 9.124 & 52.568726 & 3 & 0 & \\
\hline 03443790 & 11.840 & 12.164 & 10.602 & 1.665784 & 3 & 0 & EB Cat FP, No RV Var \\
\hline 03449540 & 14.194 & 14.533 & 12.941 & 3.212006 & 3 & 6 & \\
\hline 03458919 & 13.815 & 14.370 & 11.512 & 0.892061 & 6 & 0 & \\
\hline 03541800 & 14.367 & 14.764 & 12.994 & 4.662364 & 3 & 6 & EB Cat FP, No RV Var \\
\hline 03542573 & 12.161 & 12.613 & 10.739 & 6.942796 & 3 & 9 & \\
\hline 03556742 & 14.221 & 14.957 & 12.278 & 0.823013 & 6 & 0 & EB Cat FP, No RV Var \\
\hline 03558981 & 13.109 & 13.715 & 11.974 & 2.987858 & 6 & 6 & \\
\hline 03655326 & 14.213 & 14.614 & 12.980 & 15.066503 & 6 & 0 & \\
\hline 03656322 & 13.061 & 13.723 & 11.150 & 3.663648 & 6 & 8 & \\
\hline 03656700 & 12.997 & 13.553 & 11.398 & 0.738528 & 6 & 0 & EB Cat FP, No RV Var \\
\hline 03749508 & 13.151 & 13.536 & 11.862 & 1.065734 & 3 & 0 & \\
\hline 03765771 & 14.216 & 14.601 & 12.897 & 5.567717 & 6 & 0 & \\
\hline 03766353 & 13.968 & 14.262 & 12.744 & 2.666966 & 6 & 0 & \\
\hline 03846515 & 12.807 & 13.148 & 11.640 & 1.776084 & 3 & 0 & \\
\hline 03848919 & 13.901 & 14.477 & 12.141 & 1.047260 & 3 & 0 & \\
\hline 03848972 & 14.489 & 15.037 & 12.795 & 0.741057 & 3 & 0 & \\
\hline 03849155 & 13.831 & 14.393 & 12.271 & 1.168313 & 3 & 0 & \\
\hline 03851193 & 13.682 & 14.050 & 12.478 & 1.341079 & 3 & 7 & \\
\hline 03858804 & 13.778 & 14.501 & 11.852 & 25.951944 & 6 & 9 & EB Cat FP, No RV Var \\
\hline 03858949 & 14.576 & 15.190 & 12.872 & 25.951139 & 6 & 0 & EB Cat FP, No RV Var \\
\hline 03861595 & 11.432 & 11.755 & 10.266 & 3.849367 & 6 & 6 & \\
\hline 03867593 & 13.559 & 13.735 & 12.490 & 73.332022 & 6 & 0 & \\
\hline 03869825 & 13.320 & 13.597 & 12.180 & 4.800655 & 6 & 8 & \\
\hline 03955867 & 13.547 & 14.449 & 11.303 & 33.659962 & 3 & 8 & \\
\hline 03957477 & 12.477 & 13.001 & 10.987 & 0.979052 & 3 & 0 & \\
\hline 03964562 & 12.403 & 12.419 & 11.903 & 3.012476 & 6 & 0 & \\
\hline
\end{tabular}


Table 1

(Continued)

\begin{tabular}{|c|c|c|c|c|c|c|c|}
\hline KIC ID & $K_{p}$ & $g$ & $H$ & $P_{\text {cat }}$ & $N_{\mathrm{APG}}$ & $N_{\mathrm{HET}}$ & Notes \\
\hline 03965242 & 14.060 & 14.681 & 12.291 & 0.996722 & 6 & 0 & \\
\hline 03970233 & 14.034 & 14.656 & 12.333 & 8.254914 & 6 & 7 & \\
\hline 03971315 & 13.664 & 14.039 & 12.320 & 9.892277 & 6 & 11 & EB Cat FP, No RV Var \\
\hline 03973549 & 14.293 & 14.801 & 12.926 & 1.389955 & 6 & 0 & \\
\hline 04069063 & 13.318 & 13.733 & 11.932 & 0.504296 & 6 & 0 & \\
\hline 04069213 & 12.739 & 13.119 & 11.262 & 5.194256 & 6 & 6 & \\
\hline 04075064 & 14.951 & 15.712 & 12.904 & 61.422806 & 6 & 0 & \\
\hline 04076952 & 13.773 & 14.195 & 12.438 & 9.761169 & 3 & 8 & \\
\hline 04077442 & 13.512 & 14.348 & 11.368 & 0.692843 & 6 & 0 & \\
\hline 04078693 & 13.485 & 14.131 & 11.794 & 2.756531 & 6 & 0 & \\
\hline 04157488 & 13.961 & 14.379 & 12.467 & 5.197420 & 3 & 6 & \\
\hline 04165960 & 13.889 & 14.196 & 12.664 & 13.549178 & 6 & 0 & \\
\hline 04178389 & 14.228 & 14.695 & 12.708 & 23.210523 & 6 & 10 & \\
\hline 04275328 & 13.303 & 13.607 & 11.976 & 6.150530 & 6 & 6 & EB Cat FP, No RV Var \\
\hline 04281895 & 12.256 & 12.758 & 10.650 & 9.543588 & 6 & 8 & \\
\hline 04285087 & 12.785 & 13.188 & 11.397 & 4.486031 & 6 & 8 & \\
\hline 04372379 & 13.810 & 14.091 & 12.689 & 4.535183 & 6 & 7 & \\
\hline 04376644 & 13.767 & 14.193 & 12.371 & 27.677704 & 6 & 6 & \\
\hline 04473933 & 12.030 & 12.868 & 9.237 & 103.592625 & 6 & 8 & \\
\hline 04477830 & 13.548 & 13.894 & 12.336 & 3.384909 & 6 & 6 & EB Cat FP, No RV Var \\
\hline 04484356 & 14.235 & 14.872 & 12.359 & 1.144160 & 6 & 0 & \\
\hline 04570555 & 11.540 & 12.312 & 9.483 & 4.750303 & 6 & 7 & EB Cat FP, No RV Var \\
\hline 04570949 & 13.308 & 13.644 & 12.134 & 1.544929 & 6 & 0 & \\
\hline 04660997 & 12.317 & 12.778 & 10.763 & 0.562561 & 6 & 0 & \\
\hline 04665989 & 13.016 & 13.215 & 12.000 & 2.248067 & 6 & 0 & \\
\hline 04671584 & 13.742 & 14.137 & 12.346 & 5.593325 & 6 & 6 & \\
\hline 04672010 & 14.602 & 15.530 & 12.445 & 0.963042 & 6 & 0 & \\
\hline 04753561 & 14.928 & 15.918 & 12.647 & 4.944922 & 6 & 0 & EB Cat FP, No RV Var \\
\hline 04758368 & 10.805 & 11.670 & 8.516 & 3.749954 & 6 & 12 & \\
\hline 04840327 & 12.688 & 13.077 & 11.256 & 26.737133 & 6 & 8 & \\
\hline 04847832 & 12.450 & 13.202 & 11.051 & 30.960237 & 6 & 6 & \\
\hline 04850874 & 12.228 & 12.379 & 11.037 & 1.775906 & 6 & 0 & \\
\hline 04851217 & 11.108 & 11.316 & 10.282 & 2.470280 & 6 & 0 & \\
\hline 04931073 & 11.957 & 12.179 & 10.842 & 26.951236 & 6 & 9 & \\
\hline 04932691 & 13.627 & 13.815 & 12.641 & 18.112079 & 6 & 0 & \\
\hline 05017058 & 13.140 & 13.559 & 11.833 & 2.323895 & 6 & 0 & \\
\hline 05025294 & 13.266 & 13.704 & 11.795 & 5.462690 & 6 & 7 & \\
\hline 05193386 & 13.998 & 14.703 & 12.054 & 21.378294 & 6 & 8 & \\
\hline 05199426 & 14.080 & 14.558 & 12.532 & 78.604362 & 6 & 6 & \\
\hline 05284133 & 12.444 & 12.501 & 11.773 & 8.784576 & 6 & 5 & \\
\hline 05285607 & 11.419 & 11.684 & 10.304 & 3.899401 & 6 & 7 & \\
\hline 05288543 & 13.585 & 13.584 & 12.113 & 3.457076 & 6 & 7 & \\
\hline 05376836 & 14.041 & 14.516 & 12.571 & 3.479425 & 6 & 0 & \\
\hline 05460835 & 14.293 & 14.720 & 12.855 & 21.539274 & 6 & 0 & \\
\hline 05462901 & 15.953 & 17.140 & 13.201 & 5.270726 & 6 & 0 & \\
\hline
\end{tabular}

targets over the telescope's 30 diameter field of view, which is also fortuitously the approximate size of each Kepler module. This multiplexing capability allows us to efficiently observe many EBs with a single integration, also referred to as a visit. Each visit typically comprises eight consecutive eight-minute exposures, which are later combined by the APOGEE data reduction pipeline (Nidever et al. 2015) to produce a single visit spectrum with a total integration time of slightly more than one hour. All analysis described in this paper utilizes APOGEE data at the visit level of processing.

Our ancillary APOGEE program included two fields overlapping Kepler modules centered on the open clusters NGC 6791 (three visits) and NGC 6819 (six visits). We observed a total of 42 and 67 detached EBs in each field, respectively. All spectra for this program were obtained in 2011, during the first year of survey operations, and have been publicly released as part of SDSS Data Release 10 (Ahn et al. 2013).

Prior to analyzing the APOGEE spectra, we perform additional post-processing beyond that provided by the APOGEE pipeline. We use a low-order polynomial to remove continuum and normalize each spectrum. Residuals caused by imperfect correction of telluric absorption and sky emission are present in most pipeline-reduced APOGEE spectra; we manually correct these by interpolating over neighboring pixels. The pipeline version used for the DR10 release flagged wavelengths with suspected bad pixels by setting their flux to zero. This flagging complicates our RV measurements because a cross-correlation analysis interprets such pixels as having strong, discrete signal that is not present in correlation templates, which reduces the overall amplitude of a real 
correlation signal. We interpolate over these regions to minimize their impact.

\subsection{The HET High-resolution Spectrograph}

The small number of visits obtained by APOGEE in each of our fields are, by themselves, inadequate for deriving stellar mass at the $3 \%$ level for most of our EBs, but the infrared bandwidth provides essential leverage on the low-mass companions in high-contrast systems. Consequently, for 55 of our EBs (Table 1, column 7) we supplemented the APOGEE spectroscopy with optical spectroscopy from the $9.2 \mathrm{~m}$ HobbyEberly Telescope (hereafter HET) to achieve higher precision in spectroscopic orbital determination for the primary star. The High-resolution Spectrograph (Tull 1998, hereafter HRS) is a visible-light, fiber-fed, cross-dispersed, echelle spectrometer located on the HET (Ramsey et al. 1998). The HET was designed to carry out narrow-field spectroscopy of faint objects, and so is well suited for targets such as our Kepler EB sample. The spectrograph is housed in an isolated enclosure in the HET spectrograph room, which provides a moderately stable environment capable of achieving long-term RV stability of $25 \mathrm{~m} \mathrm{~s}^{-1}$ on bright, low-mass, main-sequence stars using standard ThAr emission lamps for wavelength calibration (Bender et al. 2012). The HET operates under a queue-based observing scheme (Shetrone et al. 2007) that allows us to request observations of a target to occur within a narrow time window. This capability allowed us to efficiently observe 55 members of our EB sample from 2011 to 2013, while targeting each system at specific orbital phases. A similar set of observations could not easily be obtained at a classically scheduled facility, making the HET a unique resource for studying binary stars.

We use the HRS with a $2^{\prime \prime}$ fiber in a configuration that provides a spectral resolution of $\lambda / \Delta \lambda=30,000$ over a bandwidth from 4076 to $7838 \AA$, except for a small gap at $5936 \AA$ where light falls between the two HRS CCDs, distributed in 73 spectral orders. This configuration provides a large number of these features, from which we derive precise radial velocities. The HET queue-based observing mode allows for the spectrograph configuration to change multiple times throughout a single night as targeting requests from different observers are carried out. Standard sequences of calibration frames, including biases, flats, and ThAr wavelength references, are typically obtained at the end of the observing session. The echelle and cross-disperser positions are not precisely repeatable at the sub-pixel level, so the standard HET operations introduce a discontinuity between the target and the ThAr observations, which can result in RV shifts of several hundred $\mathrm{m} \mathrm{s}^{-1}$. To avoid this, we obtain additional ThAr frames immediately before or after each target observation, without altering the instrument configuration. We do not obtain extra flats because the telescope overhead would be severe and our experience has shown that the misalignment inherent in the standard queue procedures is usually small enough to prevent fringing in reduced images.

To efficiently and uniformly reduce the large HRS data set generated by our Kepler EB program, we created a semiautomated data handling pipeline for the HRS. This pipeline is written in the Interactive Data Language (IDL) and carries out image processing, spectral extraction, and wavelength calibration tasks. The Appendix gives a detailed description of the pipeline.
As with the APOGEE spectra, we apply several postprocessing steps to our extracted HRS spectra prior to analysis. Each spectral order is continuum-normalized. Strong telluric contamination is mostly restricted to isolated regions at optical wavelengths. Rather than attempt a telluric correction across our HRS bandpass, we choose to retain only those regions of the spectrum with telluric contamination of $0.5 \%$ or smaller, modulus a continuity function that preserves large unbroken chunks of spectrum. In practice, we retain eight spectral windows: $\quad 4390-5025 \AA, \quad 5100-5410 \AA, \quad 5475-5680 \AA$, 5770-5855 А, 6020-6260 А, 6365-6430 А, 6620-6850 А, and 7450-7580 $\mathrm{A}$. Finally, sky emission lines are removed by interpolating over neighboring pixels.

\section{Analytical Techniques}

\subsection{Measurement of Radial Velocities}

All spectroscopy of unresolved binary stars contain the blended light of both the primary and secondary components. The signal-to-noise ratio $(\mathrm{S} / \mathrm{N})$ and the wavelength-dependent contrast ratio of an individual spectrum dictate whether that spectrum can be solved as an SB2 or only as a single-lined spectroscopic binary (SB1). Our APOGEE spectroscopy (Section 2.2) was designed to exploit the NIR contrast advantage of EBs containing a pair of main-sequence stars with a small $q$, thereby observing the systems as SB2s. Because our target sample contains a wide range of binary types, including EBs with small $q$, EBs with equal-mass components, and EBs with one or both components evolving or evolved, the suitability of optical or NIR spectroscopy for solving each EB as an SB2 is determined case by case.

We analyze our processed APOGEE and HET spectra identically, using a combination of one-dimensional and twodimensional cross-correlation algorithms to measure RVs for the primary and secondary components of each EB. For the two-dimensional case, we have implemented the TODCOR algorithm (Zucker \& Mazeh 1994) as an interactive IDL program, SXCORR, which allows the user great flexibility in quickly optimizing the correlation templates while examining multiple epochs of spectra. SXCORR simultaneously crosscorrelates two template spectra against a target spectrum containing the blended light from a binary to disentangle the component RVs. Our SXCORR implementation of TODCOR includes the maximum-likelihood extension described by Zucker (2003), modified slightly to allow segments of spectrum with variable lengths. SXCORR automatically resamples both target and template spectra into log-lambda wavelength space (Tonry \& Davis 1979), as needed depending on the input spectra. Previous investigations have extensively described our procedures for using TODCOR techniques to measure the RVs of an SB2 (e.g., Bender \& Simon 2008; Bender et al. 2012; Lockwood et al. 2014), and we refer the interested reader to the descriptions therein. Our one-dimensional correlation analysis is the trivial simplification of the two-dimensional case.

Concurrently with our HRS EB observations, we observed an extensive spectral template library of known single dwarf stars using an identical HRS configuration. This library ranges in spectral type from early $\mathrm{F}$ through mid M. Additionally, we have supplemented this library with synthetic templates generated from the PHOENIX-based BT-Settl model grid (Allard et al. 2011). These synthetic models cover a much wider range of $T_{\text {eff }},[\mathrm{M} / \mathrm{H}]$, and $\log g$ than our observed library, although 
some demonstrate substantial discrepancies with observed spectra at high resolution (Terrien et al. 2014) that manifest in our RV analysis as reduced correlation power. To generate a template from the BT-Settl library, we convolve the raw synthetic spectrum to the proper resolution (22,500 for APOGEE, 30,000 for HRS), and resample to three pixels per resolution element. We additionally apply a suite of rotational broadening kernels generated from a four-parameter nonlinear limb-darkening model (Gray 1992; Claret et al. 2012) and the appropriate stellar parameters. We do not have an observed template library for the $H$-band APOGEE spectra, so all APOGEE RVs are measured using BT-Settl templates.

\subsection{Binary Star Modeling}

To model the Kepler light curve with APOGEE and HET RV data simultaneously, we used PHOEBE 1.0 binary star modeling software (Prša \& Zwitter 2005), which is based on the Wilson-Devinney code (Wilson \& Devinney 1971, hereafter WD). To make certain that there are no numerical or systematic artifacts arising from the choice of the legacy model, we synthesized light and RV curves using PHOEBE 2.1 (Prša et al. 2016) and found no evidence for any discrepancy that exceeds the order of data scatter. We fit the data within a Bayesian framework, by utilizing EMCEE, a PYTHON implementation of the affine invariant Markov chain Monte Carlo (MCMC) ensemble sampler proposed by Goodman \& Weare (2010) and implemented by Foreman-Mackey et al. (2013). We model the noise, instrumental variations, and stellar variations caused by spots by employing CELERITE, a Gaussian process (GP) library (Foreman-Mackey et al. 2017). Prior to modeling, we minimally prepared the data by flux-normalizing and stitching the Kepler quarters and removing any obvious spurious points. The entire process is streamlined into the pipeline that is capable of processing the data autonomously. We describe the details below.

\subsubsection{Uncertainty Determination for the Kepler Data}

As the Kepler data uncertainties are commonly underestimated, we determined the uncertainties by identifying the standard deviation of sections of the light curve. For each object we identified 10 sections that contained slowly varying instrumental noise and spots, and Gaussian noise. We determined the noise of the 10 sections individually and subsequently used the average as our uncertainty value for all points on the light curve. To ensure we did not underestimate the noise in the light-curve data, we included a Gaussian noise term in our fitting procedure, which is discussed in more detail in Section 3.2.4.

\subsubsection{The PHOEBE Model}

The PHOEBE modeling software combines the complete treatment of the Roche potential with the detailed treatment of surface and horizon effects such as limb darkening, reflection, and gravity brightening to derive an accurate model of the binary parameters. The current implementation uses the WD method of summing over the discrete trapezoidal surface elements, which cover the distorted stellar surfaces, to determine an accurate representation of the total observed flux, and consequently a complete set of stellar and orbital parameters. PHOEBE incorporates all the functionality of the WD code, but also provides an intuitive graphical user interface alongside many other improvements, including updated filters, PHOENIX model atmospheres (Husser et al. 2013), and bindings that enable interfacing between PHOEBE and PYTHON.

To decrease the computational cost of using PHOEBE with EMCEE, for each iteration we created the model light curve containing 2000 data points in phase space. We then unfolded this model light curve into time space prior to adding the noise model and determining the log-likelihood. This allowed the light curve to be computed in a relatively short amount of time, but fit the model of the light curve combined with the instrumental and stellar trends to the complete data set.

The light curve and RV data were fit simultaneously. Within our models, we fit the following parameters: inclination, $i$; eccentricity, $e$; argument of periastron, the angle from the ascending node to periastron, measured in the direction of motion, $\omega$; the primary and secondary potentials, proportional to the inverse radius, $\Omega_{1}$ and $\Omega_{2}$, respectively; third light $l 3$; gamma velocity, the motion of the center of mass of the binary, $\gamma$; mass ratio, $q$; and semimajor axis, sma. We further set the albedos (reflective properties of the stars) and gravity darkening exponents (which relate to the change in temperature of the stars due to their obliquity), to the theoretical values of $A=0.6$ (Ruciński 1969b) and $\beta=0.32$ (Lucy 1967) for stars with convective envelopes $\left(T_{\text {eff }}<7000 \mathrm{~K}\right)$ and to $A=1.0$ (Ruciński 1969a) and $\beta=1.0$ (von Zeipel 1924) for stars with radiative envelopes $\left(T_{\text {eff }}>7000 \mathrm{~K}\right)$. As Diaz-Cordoves \& Gimenez (1992) showed that the square-root limb-darkening model is preferable for objects that radiate toward the IR, we applied the square-root limb-darkening law to our models and updated the limb-darkening coefficients after each iteration. Limb darkening is a thorny issue, with its implications discussed in detail in Prša et al. (2016); it affects eclipse ingress and egress and is degenerate with stellar radii. We use limb-darkening coefficients computed from the PHOENIX model atmospheres for the range of temperatures, surface gravities, and chemical abundances applicable to our systems. We enforced consistency by interpolating the limb-darkening coefficients for any explored combination of atmosphere parameters. While there are inherent limitations to using the square-root limb-darkening model, the compounded systematic effects that arise from its use (compared to other limbdarkening models) are below $0.5 \%$.

\subsubsection{MCMC Derived Uncertainties}

Objective comparison between masses and radii measured observationally and those predicted by theory requires that realistic uncertainties be derived for the measurements. We use an MCMC sampler to sample the posterior probability distribution function. At each iteration we compute the posterior log-probability distribution function:

$$
\log P(\theta \mid D)=\log P(F \mid \theta)+\log P(\mathrm{RV} \mid \theta)+\log P(\theta)+C,
$$

where $D$ denotes the data, $F$ are the light-curve measurements, $\mathrm{RV}$ are the RV measurements, $\theta$ is the parameter vector that contains the fitted parameters (specified in Section 3.2.2), and $C$ is an arbitrary constant. We incorporate EMCEE into our analysis to sample the probability distribution function within a Bayesian framework. A significant advantage of this is that the results are presented as posterior probability distribution 
functions, which indicate parameter correlations and provide more robust uncertainty estimates.

MCMC explores the parameter space using a set of walkers, in our case 142. These chains begin with a uniform prior probability distribution for each parameter. At every iteration, each chain assesses its likelihood with respect to that of another chain and then elects whether to move toward that chain. The new parameters are based on the covariance matrix of the two chains. If the move increases the posterior likelihood then it is accepted; if the move decreases it then it may be accepted with a certain probability. During the initial burn-in time, Markov chains are converging toward their maximum-likelihood position. The statistics of a large number of iterations provide converged posterior distributions for the model parameters.

We create a model of all available data (light curves and radial velocities) for each binary star. To assess convergence, we use autocorrelation timescales. The autocorrelation timescale is used to estimate the number of iterations required to generate an independent sample, i.e., the number of iterations required for the chain to "forget" where it started. We require a minimum of 30 autocorrelation timescales to achieve convergence.

Eclipse depths are related to the temperature ratio. To obtain an accurate uncertainty for the temperature ratios, and thus secondary component temperatures, we marginalized over the primary and secondary effective temperatures, and report the posterior probability of the temperature ratio. From this, we calculate the secondary temperature and its uncertainty. The minimal rotational $\mathrm{RV}$ that can be derived from APOGEE spectra is limited by the resolving power, allowing us to measure $v_{\text {rot }} \gtrsim 10 \mathrm{~km} \mathrm{~s}^{-1}$. We do not detect any broadening for any component in the studied systems, implying that all measured rotational velocities are below the $10 \mathrm{~km} \mathrm{~s}^{-1}$ detection threshold.

\subsubsection{GP Regression}

A GP is used to model noise, both instrumental and astrophysical; it is defined as a collection of random variables for which any finite number have a Gaussian distribution with a specified covariance structure. When using GPs, we use the data to condition the GP prior so that the GPs are normally distributed with respect to the data. A significant advantage of GP modeling is that it handles correlations in the data that are poorly understood by specifying only the high-level properties of a covariance kernel. We elected to use GPs to address the issues associated with stellar variations due to spots, correlated noise, and instrumental systematics that are present in the light curves for both objects. We further incorporated a white-noise kernel to assess our computed uncertainties.

Prior to the application of GPs, we create a combined binary starlight and RV curve model using PHOEBE and EMCEE. We then apply GPs to the light-curve model, which removes the need to associate the systematics in the light curve with an explicit functional form. That way, we are able to model a wide range of systematics with a small number of tunable parameters.

The kernel, or covariance function, describes the similarity between two adjacent data points. For our kernel, we elected to use a term that approximates a Matern $3 / 2$ function, which has a slowly varying component and a rapidly varying one:

$$
k(\tau)=\sigma^{2}\left[(1+1 / \epsilon) e^{-(1-\epsilon) \sqrt{3} \tau / \rho}(1+1 / \epsilon) e^{-(1+\epsilon) \sqrt{3} \tau / \rho}\right] .
$$

The parameter $\epsilon$ controls the quality of the approximation since, in the limit as $\epsilon \rightarrow 0$, this becomes the Matern 3/2 function. For our computations, we set $\epsilon$ to 0.01 . The Matern $3 / 2$ function was selected because it is capable of modeling the noise, stellar variations, and instrumental systematics in the light curve using only two parameters, $\sigma$ and $\rho$.

We additionally incorporated a white-noise term into our model. The purpose of this term is to ensure that our uncertainties are well estimated. Poorly estimated uncertainties lead to higher values for the jitter term (high in relation to the associated noise level) and the addition of white noise of the form

$$
k\left(\tau_{n, m}\right)=\sigma^{2} \delta_{n, m},
$$

where $\sigma$ is a tunable parameter.

\section{Masses and Radii for Two Example Kepler EBs}

Here we describe the results for KIC 2445134 and KIC 3003991 to demonstrate the pipeline introduced in Section 3. These objects were selected because they are total eclipsers, have small $q$, and the data are adequate for us to derive precise masses and radii. We also include the effective temperatures and metallicities as derived from the SDSS DR14 (Abolfathi et al. 2018) ASPCAP (García Pérez et al. 2016) pipeline. Although ASPCAP is optimized for giant and subgiant spectra, independent tests (Wilson et al. 2018; Andrews et al. 2019) have shown that offsets between ASPCAP temperatures and metallicities for FGK dwarfs are typically well within the ASPCAP uncertainties for our targets. Binary spectra if treated as single-component can result in significant systematic bias in ASPCAP parameters (El-Badry et al. 2018), but the effects are minimal below mass ratios of $q \sim 0.4$, where our two systems lie.

\subsection{KIC 2445134}

KIC 2445134 comprises an F-type dwarf primary and an M-type secondary orbiting with a period of 8.4 days, and has a flux ratio of $\sim 0.01$ in the Kepler bandpass. It has a Kepler magnitude of 13.55 and an $H$-band magnitude of 12.40 . The ASPCAP $T_{\text {eff }}$ is $6260 \pm 170 \mathrm{~K}$ and the metallicity is [M/ $\mathrm{H}]=-0.046 \pm 0.105$. We obtained six observations of KIC 2445134 with the HRS, which we solved as an SB1 using a mid-F-type HRS template, and three observations with APOGEE, which we solved as an SB2 using the BT-Settl templates. These measurements constrained the spectroscopic orbital parameters and allowed us to re-examine our HRS cross-correlation functions and detect the faint companion in two epochs using an HRS template for a mid M star and with correlation power slightly above the noise. Table 2 lists the date and corresponding barycentric Julian Date for each of our observations, the RVs we measure, and the associated spectrograph. Figure 1 shows the BT-Settl models corresponding to the primary and secondary components, along with the flux ratio for a synthetic EB constructed from these models. We carried out the MCMC analyses described in Section 3.2.3 on the RVs in Table 2 and the Kepler photometry, and derived 
Table 2

HRS and APOGEE RV Measurements of KIC 2445134

\begin{tabular}{|c|c|c|c|c|}
\hline UT Date & BJD - 2,400,000 & $\begin{array}{c}V_{A} \\
\left(\mathrm{~km} \mathrm{~s}^{-1}\right)\end{array}$ & $\begin{array}{c}V_{B} \\
\left(\mathrm{~km} \mathrm{~s}^{-1}\right)\end{array}$ & Instrument \\
\hline 2011 Aug 27 & 55800.804144 & $9.614 \pm 0.0450$ & $\ldots$ & HRS \\
\hline 2011 Sep 6 & 55810.756457 & $48.652 \pm 0.056$ & $-45.01 \pm 3.24$ & HRS \\
\hline 2011 Sep 24 & 55828.729442 & $57.916 \pm 0.058$ & $-68.20 \pm 3.66$ & HRS \\
\hline 2011 Oct 21 & 55855.631824 & $28.320 \pm 0.050$ & $\cdots$ & HRS \\
\hline 2011 Oct 29 & 55863.602364 & $39.702 \pm 0.056$ & $\cdots$ & HRS \\
\hline 2011 Oct 17 & 55851.578523 & $17.48 \pm 0.200$ & $28.34 \pm 0.81$ & APOGEE \\
\hline
\end{tabular}

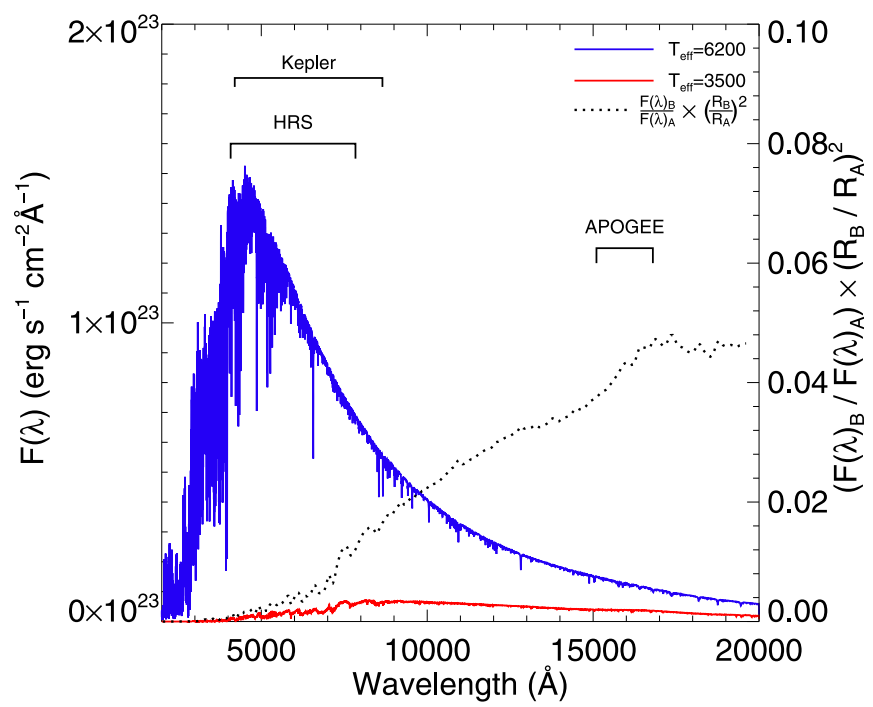

Figure 1. BT-Settl models corresponding to the components in KIC 2445134 (solid blue and red lines), and the flux ratio (dashed line). The Kepler, HRS, and APOGEE bandpasses are indicated for reference. The model spectra have been degraded to a spectral resolution of $R=2000$, while the flux ratio is shown for $R=100$. The contrast is an order of magnitude more favorable for detecting the secondary in the $H$-band than in the optical.

the orbital and physical parameters listed in Table 3. Figures 2 and 3 depict the models for the light curves and RV curves, respectively. Due to the large mass ratio, the temperature ratio, and the orientation of the orbit, the light curve of KIC 2445134 contains Doppler boosting. We incorporated Doppler boosting into our model using the framework of Bloemen et al. (2011) and present the Doppler boosting parameters for the primary and secondary components, $B_{A}$ and $B_{B}$, respectively, in Table 3. Our measurement of the mass ratio, $q=0.411 \pm 0.001$, has a precision of better than $1 \%$. Our derived masses $\left(M_{A}=1.29 \pm 0.03 M_{\odot}\right.$, $\left.M_{B}=0.53 \pm 0.01 M_{\odot}\right)$ and $\operatorname{radii}\left(R_{A}=1.42 \pm 0.01 R_{\odot}\right.$, $R_{B}=0.510 \pm 0.004 R_{\odot}$ ) have measurement precisions of $1 \%$ or better, except for the primary mass, which is constrained to $2 \%$.

To assess the dependence of the parameter uncertainties on our light-curve per-point uncertainties, we ran our software in the same manner as outlined above, but with the uncertainties multiplied by 0.5 and 2 for KIC 2445134. The outcome was that all fundamental parameters remained within the $1 \sigma$ uncertainties quoted, and the uncertainties were unchanged with the exception of the secondary radius uncertainty, which changed from $0.004 R_{\odot}$ to $0.005 R_{\odot}$ (still providing a $1 \%$
Table 3

KIC 2445134 Orbital and Physical Parameters

\begin{tabular}{|c|c|}
\hline Parameter & Value \\
\hline \multicolumn{2}{|c|}{ Derived Orbital Parameters } \\
\hline$P$ (days) & $8.41201 \pm 0.00077$ \\
\hline$T_{\text {transit }}$ & 2454972.647749 \\
\hline$i(\operatorname{deg})$ & $88.032 \pm 0.001$ \\
\hline$e$ & $0.00555 \pm 0.00001$ \\
\hline$\omega(\mathrm{rad})$ & $4.799 \pm 0.001$ \\
\hline$\Omega_{A}$ & $15.433 \pm 0.002$ \\
\hline$\Omega_{B}$ & $18.4 \pm 0.1$ \\
\hline $13(\%)$ & $4.0 \pm 0.1$ \\
\hline$T_{\text {peri. }}$ & $2455826.85 \pm 0.25$ \\
\hline$K_{A}\left(\mathrm{~km} \mathrm{~s}^{-1}\right)$ & $37.2 \pm 0.3$ \\
\hline$K_{B}\left(\mathrm{~km} \mathrm{~s}^{-1}\right)$ & $90 \pm 1$ \\
\hline$\gamma\left(\mathrm{km} \mathrm{s}^{-1}\right)$ & $21.6 \pm 0.3$ \\
\hline$q$ & $0.411 \pm 0.001$ \\
\hline $\operatorname{sma}\left(R_{\odot}\right)$ & $21.3 \pm 0.2$ \\
\hline$B_{A}$ & $3.873 \pm 0.002$ \\
\hline$B_{B}$ & $6.098 \pm 0.006$ \\
\hline \multicolumn{2}{|c|}{ Physical Parameters } \\
\hline$M_{A}\left(M_{\odot}\right)$ & $1.29 \pm 0.03$ \\
\hline$M_{B}\left(M_{\odot}\right)$ & $0.53 \pm 0.01$ \\
\hline$R_{A}\left(R_{\odot}\right)$ & $1.42 \pm 0.01$ \\
\hline$R_{B}\left(R_{\odot}\right)$ & $0.510 \pm 0.004$ \\
\hline$T_{B} / T_{A}$ & $0.635 \pm 0.001$ \\
\hline$T_{\text {eff } B}(\mathrm{~K})$ & $3976 \pm 170$ \\
\hline \multicolumn{2}{|c|}{ Gaussian Process Parameters } \\
\hline $\log \left(\phi_{\text {Matern }}\right)$ & $-0.91 \pm 0.05$ \\
\hline $\log \left(\rho_{\text {Matern }}\right)$ & $5.09 \pm 0.07$ \\
\hline $\log \left(\phi_{\text {jitter }}\right)$ & $-9.992 \pm 0.004$ \\
\hline
\end{tabular}

uncertainty) for the case of the increased per-point uncertainties.

\subsection{KIC 3003991}

KIC 3003991 represents the faint end of our EB sample, with Kepler mag $=13.9$, and comprises a late $\mathrm{G}$ star and a mid M star in a 7.2 day orbit with a Kepler flux ratio of $\sim 0.005$. The ASPCAP $T_{\text {eff }}$ is $5340 \pm 140 \mathrm{~K}$ and the metallicity is [M/ $\mathrm{H}]=-0.41 \pm 0.073$. We obtained six observations with HRS and six observations with APOGEE. Once again, we were able to easily detect the low-mass companion in the $H$-band spectra, with $\alpha \sim 0.10$, and used the measurement of $K_{B}$ from the APOGEE spectra to reanalyze the HRS spectra and recover the companion RV for many of the HRS epochs. The relative faintness of this $\mathrm{EB}$ resulted in a poorer $\mathrm{S} / \mathrm{N}$ than for $\mathrm{KIC}$ 


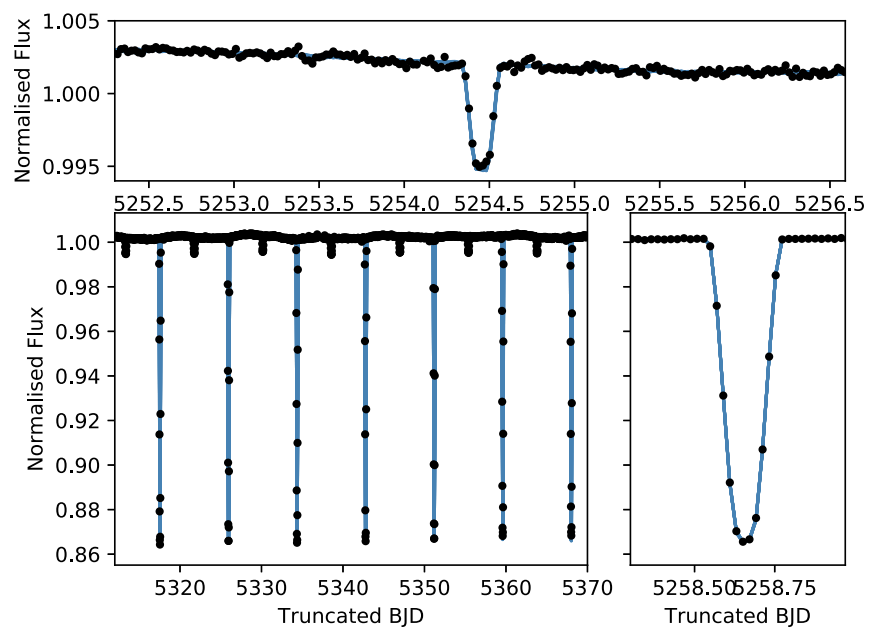

Figure 2. Kepler light curve (black) and ten models including GPs (blue) for KIC 2445134, where the different panels emphasize different regions of the light curve.

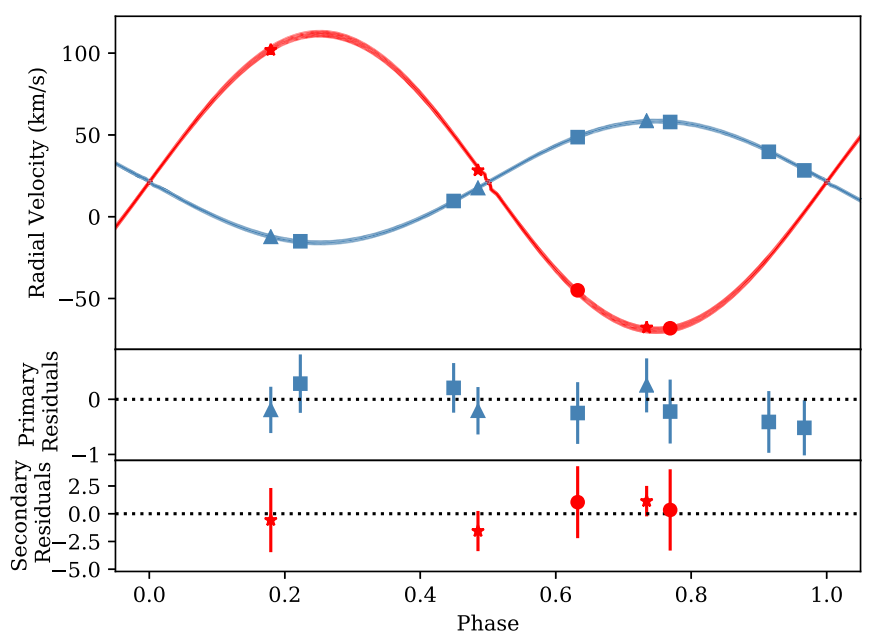

Figure 3. Phase-folded RVs for KIC 2445134 derived from the HET spectra (squares and circles) and the APOGEE spectra (triangles and stars) for the primary and secondary components, respectively. The upper panel depicts the radial velocities and ten models from the final iteration (where each model is depicted by a red or blue line). The middle and lower panels depict the residuals from the average model fit to the primary and secondary radial velocity data, respectively. The residuals are measured in $\mathrm{km} \mathrm{s}^{-1}$.

2445134 for both the APOGEE and HRS spectroscopy, which manifests as larger uncertainties on primary and secondary RVs. Table 4 lists the date and corresponding barycentric Julian Date for each of our observations, the RVs we measure, and the associated spectrograph. Figures 4 and 5 depict the light-curve model and phase-folded spectroscopic orbit for KIC 3003991, corresponding to the orbital and physical parameters in Table 5. Figure 6 shows the posterior distributions and Figure 7 shows distributions of quantities calculated from our model posteriors (where the calculations were performed after each iteration). We measure a mass ratio of $q=0.289 \pm 0.006, \quad$ and derive masses of $M_{A}=0.74 \pm 0.04 M_{\odot} \quad$ and $\quad M_{B}=0.222 \pm 0.007 M_{\odot}$, corresponding to precisions of $5 \%$ and $3 \%$, respectively. The uncertainty on both is driven largely by the uncertainty on the semi-amplitude, which is difficult to reduce further without a significant additional spectroscopic investment. As the precision we derive for $M_{B}$ complies with our program objective of
$3 \%$, it will provide useful constraints on stellar models due to the paucity of precisely measured masses in the $M \lesssim 0.25 M_{\odot}$ regime. Additionally, the radii we measure for this $\mathrm{EB}$, $R_{A}=0.84 \pm 0.01 R_{\odot}$ and $R_{B}=0.250 \pm 0.004 R_{\odot}$, have precisions of $1 \%$ and $2 \%$, respectively.

\section{False Positives in our Sample}

We selected our EB sample (Table 1) in 2011, while the analysis pipeline being used by the Kepler team to identify EBs and substellar companions was still being refined. In a few cases, the eclipse signal detected in Kepler photometry results from a background EB that is spatially unresolved from the primary KIC target at the Kepler plate scale (Abdul-Masih et al. 2016). Many of these "false positives" have subsequently been identified by the Kepler team using advanced light-curve analysis of the photometry at the pixel level, and have been flagged in the Kepler EB Catalog. We included 14 stars now labeled as false positives for which we have APOGEE or HRS spectra to look for RV variability. In all cases, these targets show no variability within the RV measurement precisions listed in Section 2. These targets, which we have dynamically verified as false positives, are identified in the last column of Table 1. Figure 8 illustrates the attained RV precision of a star (top panel) that does not have a stellar component with a period within our baseline, amounting to better than $\sim 100 \mathrm{~m} \mathrm{~s}^{-1}$. The magnitude and RV precision of this star are representative of stars observed from this program with the HET. The RV measurement precision of a false positive observed with APOGEE is also shown (bottom panel), and is representative of the typical RV uncertainty for our APOGEE targets.

\section{Discussion and Future Prospects}

The two EBs presented in Section 4 illustrate the contributions made by our ongoing work to the direct and precise measurement of masses and radii for low-mass stars. Many systems in our EB sample have primary components with mass $M>0.8 M_{\odot}$, a regime where models of stellar mass and radii are well matched to observational constraints. Many have small mass ratios $(q<0.6)$, and so have low-mass secondaries, specifically targeting the regime with disagreement between models and existing observations. The high-mass primary allows traditional spectroscopic analysis tools to obtain metallicity for the spectra since the secondary star flux is low enough that the effect of spectral contamination is minimal. As discussed in Terrien et al. (2012) the stellar metallicity is an important constraint on the models, as well as being necessary to derive ages. This project aims to derive masses and radii for low-mass stars using a combination of precision photometry from Kepler coupled with radial velocities from stable fiber-fed optical and near-infrared high-resolution spectrographs. Figure 9 depicts the mass-radius relationship for the stars in this work (filled blue and black circles), compared with those from DEBCat (open circles). Figure 10 depicts the same but with $[\mathrm{Fe} / \mathrm{H}]=-1$ isochrones (as opposed to solar metallicity). The primary and secondary components of KIC 2445134 both show good agreement (within $1 \sigma$ ) with the $2 \mathrm{Gyr}$ solar metallicity isochrones. The primary component of KIC 3003991 shows good agreement (within $1 \sigma$ ) with the $10 \mathrm{Gyr}$ isochrones, but the secondary component is $3 \sigma$ away. This kind of disagreement is not unusual in the literature for low-mass stars (Torres et al. 2010). Our program with 
Table 4

HRS and APOGEE RV Measurements of KIC 3003991

\begin{tabular}{|c|c|c|c|c|}
\hline UT Date & BJD - 2,400,000 & $\begin{array}{c}V_{A} \\
\left(\mathrm{~km} \mathrm{~s}^{-1}\right)\end{array}$ & $\begin{array}{c}V_{B} \\
\left(\mathrm{~km} \mathrm{~s}^{-1}\right)\end{array}$ & Instrument \\
\hline 2011 Oct 8 & 55842.687885 & $-144.119 \pm 0.072$ & $-50.68 \pm 8.35$ & HRS \\
\hline 2012 Mar 21 & 56007.990683 & $-120.157 \pm 0.065$ & $\cdots$ & HRS \\
\hline 2012 May 28 & 56075.798304 & $-143.297 \pm 0.061$ & $-50.92 \pm 8.21$ & HRS \\
\hline 2012 Jun 8 & 56086.797501 & $-103.329 \pm 0.064$ & $-185.18 \pm 12.08$ & HRS \\
\hline 2012 Jun 10 & 56088.766870 & $-140.972 \pm 0.06$ & $-54.93 \pm 5.63$ & HRS \\
\hline 2011 Oct 6 & 55840.6597900 & $-106.210 \pm 0.310$ & $-165.85 \pm 6.30$ & APOGEE \\
\hline 2011 Oct 15 & 55849.5774200 & $-139.530 \pm 0.200$ & $-69.54 \pm 3.22$ & APOGEE \\
\hline 2011 Oct 17 & 55851.6479100 & $-136.690 \pm 0.170$ & $-72.76 \pm 3.51$ & APOGEE \\
\hline 2011 Nov 1 & 55866.5693400 & $-128.360 \pm 0.170$ & $-96.77 \pm 4.51$ & APOGEE \\
\hline
\end{tabular}

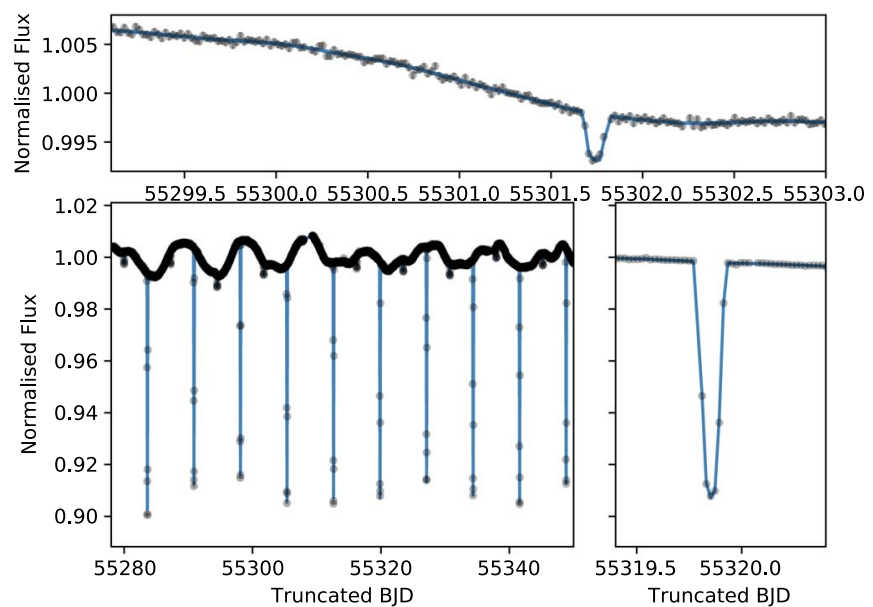

Figure 4. Kepler light curve (black) and ten models including GPs (blue) for KIC 3003991, where the different panels emphasize different regions of the light curve.

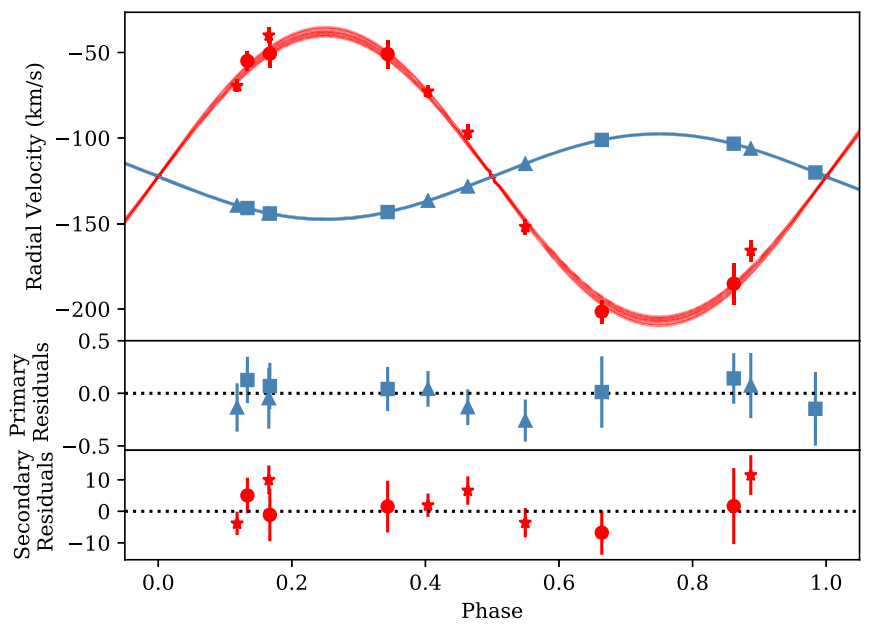

Figure 5. Phase-folded RVs for KIC 3003991. The layout is identical to Figure 3. The thickness of the lines depicts the spread of the ten models caused by the model uncertainty. This is particularly significant for the secondary component (red lines).

APOGEE will continue to fill in parameter space by obtaining double-line spectroscopic RVs for systems with high flux contrast. KIC 2445134 and KIC 3003991 would have been especially challenging without the benefit of NIR spectroscopy.
Table 5

KIC 3003991 Orbital and Physical Parameters

\begin{tabular}{|c|c|}
\hline Parameter & Value \\
\hline \multicolumn{2}{|c|}{ Derived Orbital Parameters } \\
\hline$P$ (days) & $7.24478 \pm 0.00062$ \\
\hline$T_{\text {transit }}$ & 2454964.859085 \\
\hline$i(\operatorname{deg})$ & $88.178 \pm 0.008$ \\
\hline$e$ & $0.00030 \pm 0.00003$ \\
\hline$\omega(\mathrm{rad})$ & $4.09 \pm 0.03$ \\
\hline$\Omega_{A}$ & $18.76 \pm 0.02$ \\
\hline$\Omega_{B}$ & $19.9 \pm 0.3$ \\
\hline$l 3(\%)$ & $3.5 \pm 0.2$ \\
\hline$T_{\text {peri. }}$ & $2455953.65 \pm 0.18$ \\
\hline$K_{A}\left(\mathrm{~km} \mathrm{~s}^{-1}\right)$ & $24.97 \pm 0.05$ \\
\hline$K_{B}\left(\mathrm{~km} \mathrm{~s}^{-1}\right)$ & $83 \pm 1$ \\
\hline$\gamma\left(\mathrm{km} \mathrm{s}^{-1}\right)$ & $-122.55 \pm 0.03$ \\
\hline$q$ & $0.298 \pm 0.006$ \\
\hline $\operatorname{sma}\left(R_{\odot}\right)$ & $15.6 \pm 0.2$ \\
\hline \multicolumn{2}{|c|}{ Physical Parameters } \\
\hline$M_{A}\left(M_{\odot}\right)$ & $0.74 \pm 0.04$ \\
\hline$M_{B}\left(M_{\odot}\right)$ & $0.222 \pm 0.007$ \\
\hline$R_{A}\left(R_{\odot}\right)$ & $0.84 \pm 0.01$ \\
\hline$R_{B}\left(R_{\odot}\right)$ & $0.250 \pm 0.004$ \\
\hline$T_{B} / T_{A}$ & $0.662 \pm 0.001$ \\
\hline$T_{\text {eff } B}(\mathrm{~K})$ & $3536 \pm 140$ \\
\hline \multicolumn{2}{|c|}{ Gaussian Process Parameters } \\
\hline $\log \left(\phi_{\text {Matern }}\right)$ & $-0.3 \pm 0.1$ \\
\hline $\log \left(\rho_{\text {Matern }}\right)$ & $4.3 \pm 0.1$ \\
\hline $\log \left(\phi_{\mathrm{jitter}}\right)$ & $-10.996 \pm 0.004$ \\
\hline
\end{tabular}

For these systems we succeeded in obtaining the 3\% program goal for the uncertainties on the masses and radii (with the exception of the primary mass of KIC 3003991), making these valuable benchmark systems. Additional results emerging from this program will further populate the mass-radius plane for low-mass dwarfs, study systems with equal-mass components, and provide high-precision checks on parameters derived from asteroseismology for a few, rare giants in EB systems with detected stellar oscillations.

We acknowledge support from two NASA ADAP grants (NNX13AF32G and 16-ADAP16-0201), and NSF grant AST 1517592 for this project. This work was partially supported by funding from the Center for Exoplanets and Habitable Worlds. The Center for Exoplanets and Habitable Worlds is supported 


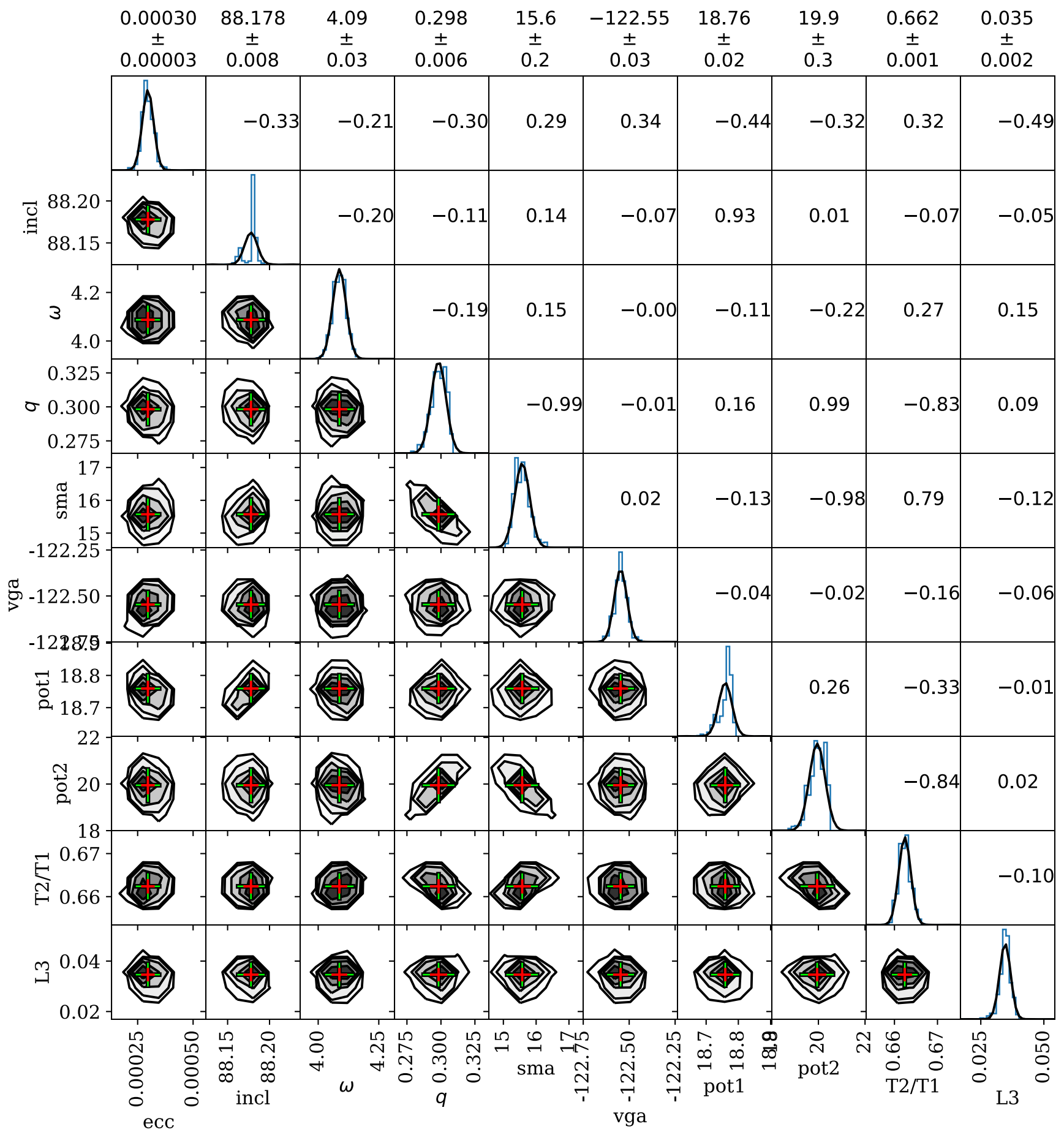

Figure 6. Posterior distributions for the KIC 3003991 parameters derived from Kepler photometry, and APOGEE and HRS radial velocities. Boxes in the upper right contain the standard correlation coefficient, with 1 corresponding to perfect correlation, -1 perfect anticorrelation, and 0 no correlation. Final parameter values are listed along the top.

by the Pennsylvania State University, the Eberly College of Science, and the Pennsylvania Space Grant Consortium. We acknowledge support from NSF grants AST 1006676 and AST 1126413 in our pursuit of precision radial velocities in the NIR. This research has made use of the SIMBAD database, operated at CDS, Strasbourg, France. This publication makes use of data products from the Two Micron All Sky Survey, which is a joint project of the University of Massachusetts and the Infrared Processing and Analysis Center/California Institute of Technology, funded by the National Aeronautics and Space
Administration and the National Science Foundation. Finally, we acknowledge the thorough review of the anonymous reviewer whose comments saved us from a small embarrassment.

This work was based on observations with the SDSS $2.5 \mathrm{~m}$ telescope. Funding for SDSS-III has been provided by the Alfred P. Sloan Foundation, the Participating Institutions, the National Science Foundation, and the U.S. Department of Energy of Science. The SDSS-III website is http://www.sdss3. org/. SDSS-III is managed by the Astrophysical Research 


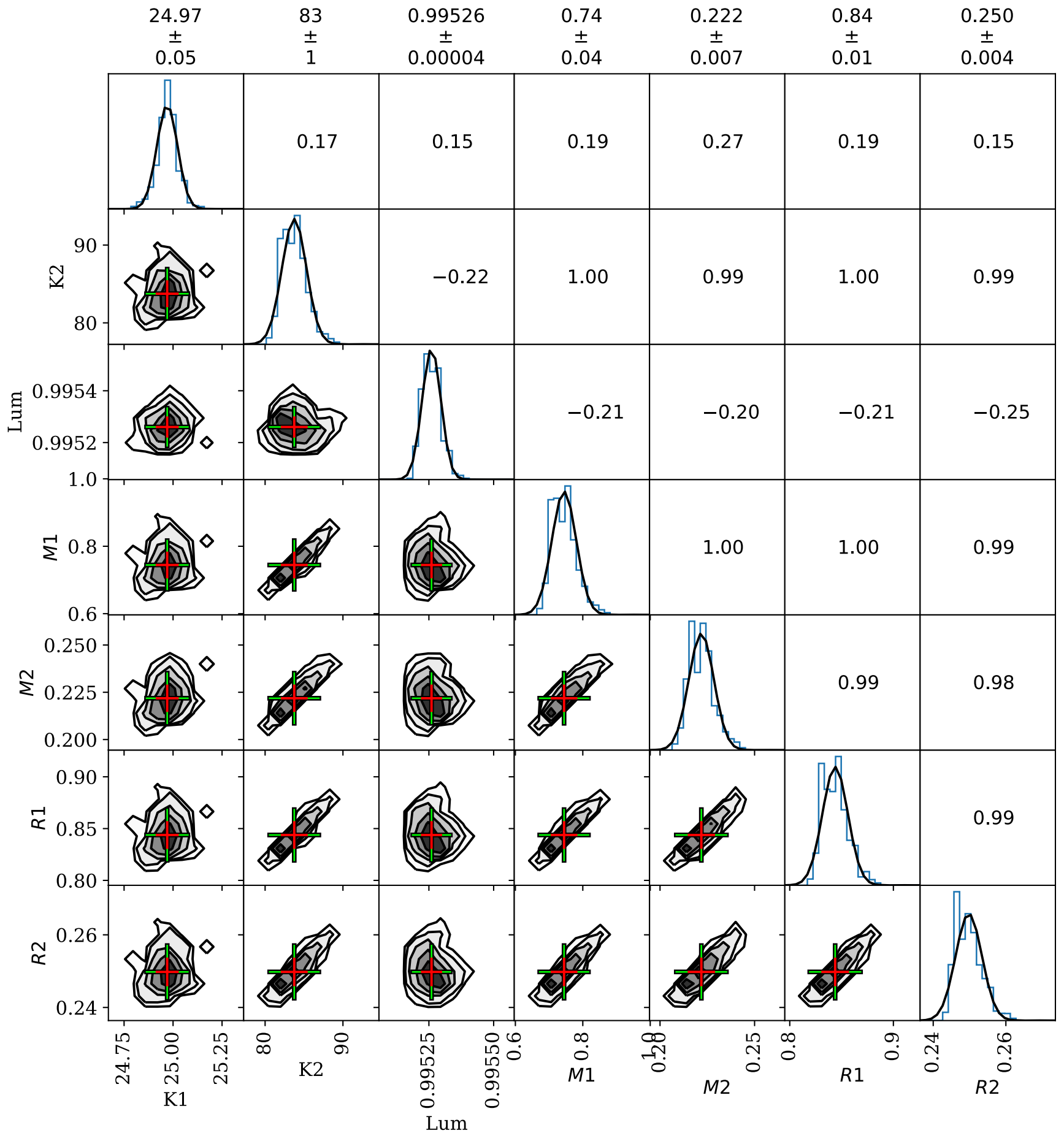

Figure 7. Parameter distributions for the calculated parameters of KIC 3003991. Layout is as in Figure 6.

Consortium for the Participating Institutions of the SDSS-III Collaboration including the University of Arizona, the Brazilian Participation Group, Brookhaven National Laboratory, University of Cambridge, Carnegie Mellon University, University of Florida, the French Participation Group, the German Participation Group, Harvard University, the Instituto de Astrofísica de Canarias, the Michigan State/Notre Dame/ JINA Participation Group, Johns Hopkins University, Lawrence Berkeley National Laboratory, Max Planck Institute for Astrophysics, Max Planck Institute for Extraterrestrial Physics, New Mexico State University, New York University, Ohio State University, Pennsylvania State University, University of
Portsmouth, Princeton University, the Spanish Participation Group, University of Tokyo, University of Utah, Vanderbilt University, University of Virginia, University of Washington, and Yale University.

Data presented herein were also obtained at the HobbyEberly Telescope (HET), a joint project of the University of Texas at Austin, the Pennsylvania State University, Stanford University, Ludwig-Maximilians-Universität München, and Georg-August-Universität Göttingen. The HET is named in honor of its principal benefactors, William P. Hobby and Robert E. Eberly. 

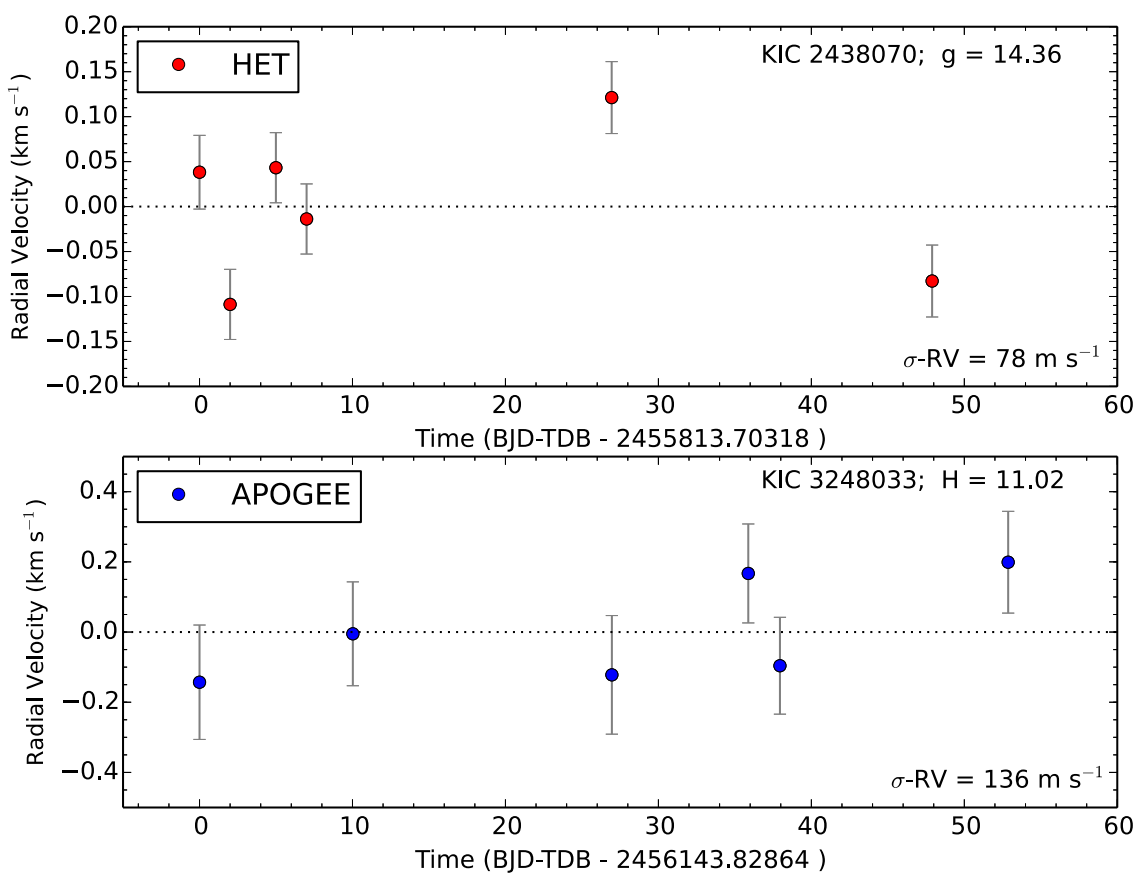

Figure 8. RV measurements of KIC 3248033 identified as a false positive based on pixel-level analysis of its photometry and of the red giant KIC 2438070 with no stellar eclipsing companion. Our RV measurements dynamically verify that the eclipse signal in the light-curve data results from an unresolved background EB which is highly diluted by the foreground KIC target.

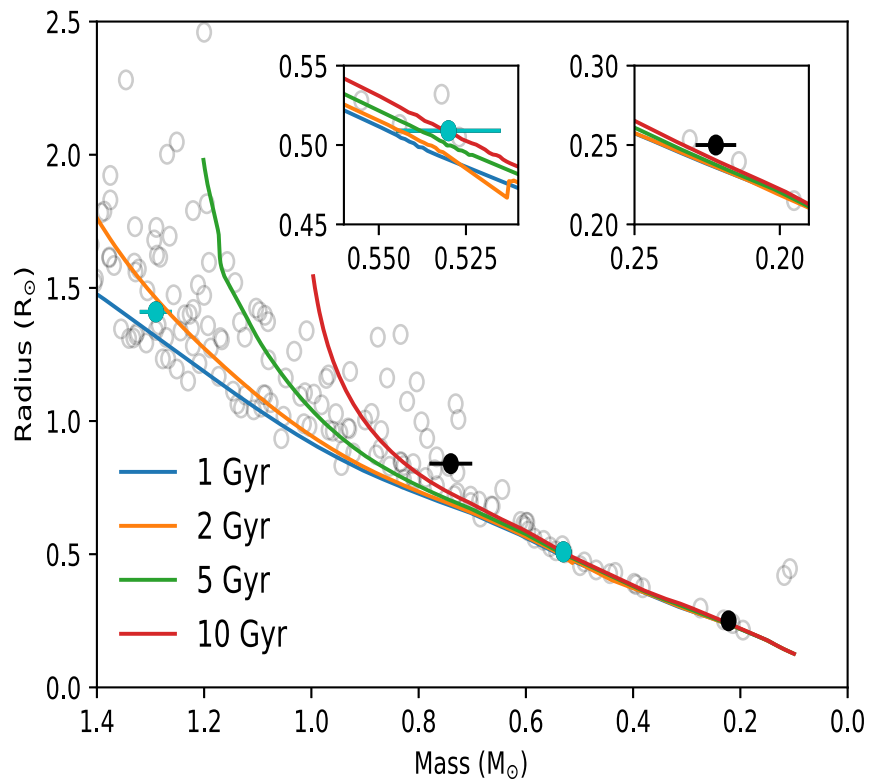

Figure 9. Radius vs. mass, plotted for the EBs in the DEBCat Eclipsing Binary Catalog (open circles), along with the two EBs we describe in Section 4, where KIC 2445134 is depicted with solid blue circles and KIC 3003991 is depicted with solid black circles. For comparison, we show MIST (MESA Isochrones and Stellar Tracks) stellar isochrones (Choi et al. 2016; Dotter 2016) for stars of solar metallicity. The insets depict magnified regions for the secondary components of KIC 2445134 (left) and KIC 3003991 (right). The paucity of precision measurements below $\sim 0.8 M_{\odot}$, and particularly below $\sim 0.25 M_{\odot}$, is evident.

\section{Appendix}

\section{Detailed Description of Our HET HRS Pipeline}

Our EB project makes use of an internal data reduction pipeline for the HRS on HET. For future reference with other papers in this series, and for others using this instrument on the HET, we provide a detailed description of our pipeline, which

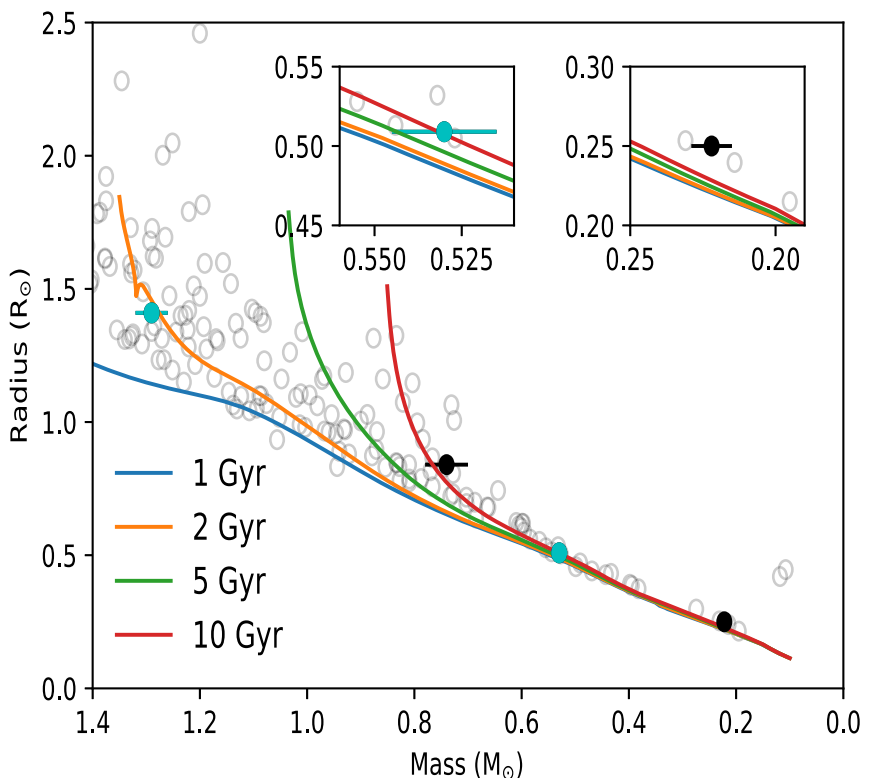

Figure 10. The same as Figure 9, but with $[\mathrm{Fe} / \mathrm{H}]=-1$. In this case, the primary component of KIC 3003991 now agrees with the $10 \mathrm{Gyr}$ isochrone, but the secondary component does not. This is in line with a known disparity between theoretical stellar models and observations in the low-mass region, and further highlights the importance of modeling low-mass binary components (Torres et al. 2010).

is composed of a series of independent subroutines that can be combined into nightly processing scripts; we refer to on-sky observations of science targets generically as target frames, and individual calibrations either generically as calibrations or by their specific function. As an aside, the pipeline can easily process HRS data taken in any of the possible HRS configurations, and so, as a service to other observers who may be interested in utilizing its functionality, the description 
we provide here is intended to be general, rather than specific to the HRS configuration listed above.

A raw HRS FITS image contains three components: the main FITS header describes the instrument configuration and target exposure, while the first and second FITS extensions contain the red and blue image data, respectively. Instrument keywords in the main header can reliably reconstruct the HRS configuration used for any given observation, without relying on external $\log$ sheets or records. Keywords describing the target, such as OBSTYPE and OBJECT, are manually specified by the HET resident astronomer carrying out the observation, and are not always used consistently. To begin reducing a night's observations, we copy the raw target and calibration images into a single working directory. The pipeline automatically classifies the various calibration and target frames, and carries out basic image processing on them. If the working directory contains images taken with different HRS configurations, each configuration will be parsed and handled automatically.

First, HRS_SPLIT extracts the red and blue images from the multi-extension FITS file, saves them as individual zeroextension FITS images with a suffix of “_r" or “_b," and assigns the data contained in the original header. This step ensures that the original FITS files are not modified in any way by the pipeline. We then perform an overscan correction (HRS_OVERSCAN) by trimming each image to the data region and subtracting a pedestal value determined by computing the median of the overscan region. Higher order corrections that could be derived from the overscan are instead corrected with the bias frames. Different HRS configurations utilize different on-chip CCD binning schemes, and HRS_BIAS automatically groups and combines bias frames based on their binning, producing master bias frames for each configuration used during a night. A similar process is then performed on all available flat fields (HRS_FLAT), although the number of possible configurations is much larger for flats than for bias frames. HRS_FLAT properly separates multiple sets of flats from the same configuration that were obtained with different exposure times, which is occasionally necessary when flats with very high signal-to-noise ratio are required. In addition, non-standard flat fields taken through the HRS target fibers illuminated by the Medium Resolution Spectrograph (Ramsey et al. 2003) flat lamp or twilight sky are properly recognized. Each master flat field is automatically bias-corrected and saved with a unique name identifying the light source, instrument configuration, and exposure time. Similar corrections are applied to individual target frames. These image processing steps can generally be carried out without any human input or supervision, and are conveniently grouped in a script, HRS_IPROC, which requires no input parameters or keywords.

Extracting one-dimensional spectra from a two-dimensional image requires locating and tracing each echelle order in the target image. The HRS dispersion runs vertically across the detector (our $y$ coordinate), and we make the simplifying (but essentially correct) assumption that the HRS slit is aligned perfectly with detector pixel rows (our $x$ coordinate). Each order is traced independently, and each trace begins by identifying one $x$ and $y$ pair that intercepts the fiber profile. At that position we extract a subarray with an $x$ dimension of 1.5 times the physical width of the fiber in pixels, which fully encloses the illuminated pixels without overlapping into the adjacent orders, and a $y$ dimension of 4 pixels, which averages over noise and increases the speed of the tracing procedure. The illumination profile of a fiber resembles a top hat, with some variation across the illuminated pixels due to imperfect radial scrambling in the fiber. This functional form is not well represented by the Gaussian profile commonly used for slit-fed spectrographs. Instead, to identify the $x$ coordinate of the beam center we compute the derivative of the profile and fit that with a combination of two Gaussians of equal width, separated by the slit width in pixels. This parameterization reliably recovers the beam center position at the selected $y$ coordinate. The algorithm then walks up and down the beam, computing $x$ for each binned $y$. We fit these measured beam positions with a low-order polynomial to derive the beam position at each dispersion position.

The tracing algorithm is implemented in two separate subroutines: HRS_MTRACE and HRS_ATRACE. HRS_MTRACE allows the user to interactively identify beams, and annotate each with the echelle order and the type of spectrum (absorption, such as a target or flat field, or emission, such as a sky or ThAr). This information is encapsulated in a template file that fully describes the HRS configuration. HRS_ATRACE is fully automated, and uses previously derived templates to process routine sets of images, retracing all beams to compensate for any misalignment between the template and the target frame due to imprecision in repositioning the HRS components.

Preserving the relative flux information in our science target frames requires that the master flat frames be normalized to remove both the echelle blaze and the fiber illumination profile. HRS_ELATNORM derives this normalization, using a procedure conceptually similar to optimal spectral extraction. Such schemes have been described extensively in the past (e.g., Horne 1986; Mukai 1990; Cushing et al. 2004), and we have adapted them for use with a fiber-fed echelle spectrograph. Each master flat is traced, and then each beam is rectified. The rectified beam is used to derive a dispersion-dependent spatial profile for the beam, following the procedure outlined by Cushing et al. (2004) and figures therein. This profile is the desired function for normalizing the flat fields. Reversing the image rectification on the beam profile yields the normalization image that can be applied to the original master flat frame.

To extract the individual target spectra, HRS_OPTEXT divides the normalized flat field into the two-dimensional target image, and then applies an optimal extraction algorithm similar to that used for normalizing the flat fields. If multiple exposures of the same target were obtained, the user has the option to extract each image individually, or coadd them in two dimensions and extract the composite spectrum. Optimal fiber profiles are automatically derived for each beam, and provide the proper weighting functions for optimal extraction. A primary benefit often associated with optimal extraction algorithms is their ability to optimize the $\mathrm{S} / \mathrm{N}$ of the extracted spectrum by minimizing the contribution of detector noise. While this is useful for some of our fainter Kepler EB targets, more useful is the algorithm's ability to automatically identify bad pixels in the two-dimensional images caused by detector defects or cosmic rays. These pixels are automatically excluded and the weighting function at each affected dispersion element is adjusted accordingly. HRS_OPTEXT returns optimally extracted spectra, sum-extracted spectra for comparison purposes, and wavelength-dependent variances that provide realistic measures of the spectrum $\mathrm{S} / \mathrm{N}$. These data products, as 
well as descriptions of each echelle order and beam, are encapsulated in a FITS table for each target image.

Our wavelength calibration pipeline relies on a construct similar to that behind the tracing algorithms: time-intensive manual line identification defines templates for each HRS configuration, and these templates are used in an automated reidentification procedure for routine data processing. The templates are generated using the IDL-based WAVECAL package (Piskunov \& Valenti 2002) to associate the wavelengths of known ThAr spectral lines with their $y$ pixel location for each spectral order. Wavelengths are taken from the linelist of Murphy et al. (2007), and typically number $\sim 10-20$ per order. The set of lines comprising each order is fit with a loworder polynomial (typically fourth order) to derive the template dispersion solution for that order. To calibrate a target spectrum, we automatically measure the ThAr image taken consecutively to the target observation, using the template as a guide. The dispersion solution for each order is automatically solved for, using a sigma-clipping rejection to detect poorly fit lines, and the resulting wavelength solution is applied to the corresponding target spectrum. Although the HRS is not a pressure- or temperature-stabilized instrument, this careful use of ThAr calibrations consecutive with target observations can yield RV stability of $25 \mathrm{~m} \mathrm{~s}^{-1}$ or better for observations taken on different nights, weeks, or even months (e.g., Bender et al. 2012).

\section{ORCID iDs}

Suvrath Mahadevan (iD https://orcid.org/0000-00019596-7983

Chad F. Bender (iD https://orcid.org/0000-0003-4384-7220

Kelly Hambleton (iD https://orcid.org/0000-0001-5473-856X

Scott W. Fleming (iD https://orcid.org/0000-0003-0556-027X

Kyle Conroy (iD https://orcid.org/0000-0002-5442-8550

Leslie Hebb (ib https://orcid.org/0000-0003-1263-8637

Arpita Roy (iD https://orcid.org/0000-0001-8127-5775

Andrej Prša (i) https://orcid.org/0000-0002-1913-0281

\section{References}

Abdul-Masih, M., Prša, A., Conroy, K., et al. 2016, AJ, 151, 101 Abolfathi, B., Aguado, D. S., Aguilar, G., et al. 2018, ApJS, 235, 42 Ahn, C. P., Alexandroff, R., Allende Prieto, C., et al. 2013, arXiv:1307.7735 Allard, F., Homeier, D., \& Freytag, B. 2011, ASP Conf. Ser. 448 in 16th

Cambridge Workshop on Cool Stars, Stellar Systems, and the Sun (San Francisco, CA: ASP), 91

Allende Prieto, C., Majewski, S. R., Schiavon, R., et al. 2008, AN, 329, 1018 Andersen, J. 1991, A\&ARv, 3, 91

Andrews, J. J., Anguiano, B., Chanamé, J., et al. 2019, ApJ, 871, 42

Bender, C. F., Mahadevan, S., Deshpande, R., et al. 2012, ApJL, 751, L31

Bender, C. F., \& Simon, M. 2008, ApJ, 689, 416

Berger, D. H., Gies, D. R., McAlister, H. A., et al. 2006, ApJ, 644, 475

Bloemen, S., Marsh, T. R., Østensen, R. H., et al. 2011, MNRAS, 410, 1787

Borucki, W. J., Koch, D., Basri, G., et al. 2010, Sci, 327, 977

Caldwell, D. A., Kolodziejczak, J. J., Van Cleve, J. E., et al. 2010, ApJL, 713, L92

Carter, J. A., Fabrycky, D. C., Ragozzine, D., et al. 2011, Sci, 331, 562

Casewell, S. L., Raynard, L., Watson, C. A., et al. 2018, MNRAS, 481, 1897

Castelli, F., \& Kurucz, R. L. 2004, arXiv:astro-ph/0405087

Chabrier, G., Gallardo, J., \& Baraffe, I. 2007, A\&A, 472, L17

Choi, J., Dotter, A., Conroy, C., et al. 2016, ApJ, 823, 102

Claret, A., Hauschildt, P. H., \& Witte, S. 2012, A\&A, 546, A14

Conroy, K. E., Prša, A., Stassun, K. G., et al. 2014, PASP, 126, 914

Cruz, P., Diaz, M., Birkby, J., et al. 2018, MNRAS, 476, 5253

Cushing, M. C., Vacca, W. D., \& Rayner, J. T. 2004, PASP, 116, 362

Diaz-Cordoves, J., \& Gimenez, A. 1992, A\&A, 259, 227

Dittmann, J. A., Irwin, J. M., Charbonneau, D., et al. 2017, ApJ, 836, 124
Dotter, A. 2016, ApJS, 222, 8

Doyle, L. R., Carter, J. A., Fabrycky, D. C., et al. 2011, Sci, 333, 1602

Eisenstein, D. J., Weinberg, D. H., Agol, E., et al. 2011, AJ, 142, 72

El-Badry, K., Rix, H.-W., Ting, Y.-S., et al. 2018, MNRAS, 473, 5043

Faigler, S., \& Mazeh, T. 2011, MNRAS, 415, 3921

Faigler, S., Mazeh, T., Quinn, S. N., Latham, D. W., \& Tal-Or, L. 2012, ApJ, 746, 185

Feiden, G. A., \& Chaboyer, B. 2013, ApJ, 779, 183

Feiden, G. A., Chaboyer, B., \& Dotter, A. 2011, ApJL, 740, L25

Foreman-Mackey, D., Agol, E., Ambikasaran, S., \& Angus, R. 2017, celerite, Astrophysics Source Code Library, ascl:1709.008

Foreman-Mackey, D., Hogg, D. W., Lang, D., \& Goodman, J. 2013, PASP, 125,306

García Pérez, A. E., Allende Prieto, C., Holtzman, J. A., et al. 2016, AJ, 151,144

Gómez Maqueo Chew, Y., Morales, J. C., Faedi, F., et al. 2014, A\&A, 572, A50

Goodman, J., \& Weare, J. 2010, Communications in Applied Mathematics and Computational Science, 5, 65

Goodricke, J. 1783, Royal Society of London Philosophical Transactions Series I, 73, 474

Gray, D. F. 1992, The Observation and Analysis of Stellar Photospheres ( ) (Cambridge: Cambridge Univ. Press)

Gunn, J. E., Siegmund, W. A., Mannery, E. J., et al. 2006, AJ, 131, 2332

Higl, J., \& Weiss, A. 2017, A\&A, 608, A62

Horne, K. 1986, PASP, 98, 609

Husser, T.-O., Wende-von Berg, S., Dreizler, S., et al. 2013, A\&A, 553, A6

Irwin, J. M., Quinn, S. N., Berta, Z. K., et al. 2011, ApJ, 742, 123

Kesseli, A. Y., Kirkpatrick, J. D., Fajardo-Acosta, S. B., et al. 2018, arXiv: 1810.07702

Kirk, B., Conroy, K., Prša, A., et al. 2016, AJ, 151, 68

Konacki, M., Muterspaugh, M. W., Kulkarni, S. R., \& Hełminiak, K. G. 2010, ApJ, 719, 1293

Kraus, A. L., Tucker, R. A., Thompson, M. I., Craine, E. R., \& Hillenbrand, L. A. 2011, ApJ, 728, 48

Lockwood, A. C., Johnson, J. A., Bender, C. F., et al. 2014, arXiv:1402.0846 López-Morales, M. 2007, ApJ, 660, 732

Lubin, J. B., Rodriguez, J. E., Zhou, G., et al. 2017, ApJ, 844, 134

Lucy, L. B. 1967, ZA, 65, 89

MacDonald, J., \& Mullan, D. J. 2017, ApJ, 850, 58

Majewski, S. R., Schiavon, R. P., Frinchaboy, P. M., et al. 2017, AJ, 154, 94

Markwardt, C. B. 2009, adass XVIII, 411, 251

Matijevič, G., Prša, A., Orosz, J. A., et al. 2012, AJ, 143, 123

Mazeh, T., Simon, M., Prato, L., Markus, B., \& Zucker, S. 2003, ApJ, 599, 1344

Mighell, K. J., \& Plavchan, P. 2013, AJ, 145, 148

Morales, J. C., Gallardo, J., Ribas, I., et al. 2010, ApJ, 718, 502

Morales, J. C., Ribas, I., \& Jordi, C. 2008, A\&A, 478, 507

Mukai, K. 1990, PASP, 102, 183

Murphy, M. T., Tzanavaris, P., Webb, J. K., \& Lovis, C. 2007, MNRAS, 378,221

Nidever, D. L., Holtzman, J. A., Allende Prieto, C., et al. 2015, AJ, 150, 173

Piskunov, N. E., \& Valenti, J. A. 2002, A\&A, 385, 1095

Planck, M. 1900a, V. Deut. Phys. Ges, 2, 202

Planck, M. 1900b, V. Deut. Phys. Ges, 2, 237

Popper, D. M. 1980, ARA\&A, 18, 115

Prato, L., Simon, M., Mazeh, T., et al. 2002, ApJ, 569, 863

Prša, A., Batalha, N., Slawson, R. W., et al. 2011, AJ, 141, 83

Prša, A., Conroy, K. E., Horvat, M., et al. 2016, ApJS, 227, 29

Prša, A., \& Harmanec, P. 2012, IAU Symp. 282 in From Interacting Binaries to Exoplanets: Essential Modeling Tools, ed. M.T. Richards \& I. Hubeny (Cambridge: Cambridge Univ. Press), 339

Prša, A., \& Zwitter, T. 2005, ApJ, 628, 426

Ramsey, L. W., Adams, M. T., Barnes, T. G., et al. 1998, Proc. SPIE, 3352, 34 Ramsey, L. W., Engel, L. G., Sessions, N., et al. 2003, Proc. SPIE, 4841, 1036 Ruciński, S. M. 1969a, AcA, 19, 125

Ruciński, S. M. 1969b, AcA, 19, 245

Schwamb, M. E., Orosz, J. A., Carter, J. A., et al. 2013, ApJ, 768, 127

Shetrone, M., Cornell, M. E., Fowler, J. R., et al. 2007, PASP, 119, 556

Slawson, R. W., Prša, A., Welsh, W. F., et al. 2011, AJ, 142, 160

Tal-Or, L., Faigler, S., \& Mazeh, T. 2015, A\&A, 580, A21

Terrien, R. C., Fleming, S. W., Mahadevan, S., et al. 2012, ApJL, 760, L9

Terrien, R. C., Mahadevan, S., Deshpande, R., et al. 2014, ApJ, 782, 61

Tonry, J., \& Davis, M. 1979, AJ, 84, 1511

Torres, G., Andersen, J., \& Giménez, A. 2010, A\&ARv, 18, 67

Tull, R. G. 1998, Proc. SPIE, 3355, 387 
von Zeipel, H. 1924, MNRAS, 84, 702

Wilson, J. C., Hearty, F., Skrutskie, M. F., et al. 2010, Proc. SPIE, 7735, $77351 \mathrm{C}$

Wilson, R. E. 1979, ApJ, 234, 1054

Wilson, R. E. 1993, ASP Conf. Ser. 38 in New frontiers in binary star research, ed. K.-C. Leung \& II-Seong Nha (San Francisco, CA: ASP), 91

Wilson, R. E. 2007, A\&AT, 26, 3

Wilson, R. E., \& Devinney, E. J. 1971, ApJ, 166, 605
Wilson, R. E., \& Wyithe, S. B. 2003, ASP Conf. Ser. 298 in GAIA Spectroscopy: Science and Technology, ed. U. Munari (San Francisco, CA: ASP), 313

Wilson, R. F., Teske, J., Majewski, S. R., et al. 2018, AJ, 155, 68

York, D. G., Adelman, J., Anderson, J. E., Jr., et al. 2000, AJ, 120, 1579

Zasowski, G., Johnson, J. A., Frinchaboy, P. M., et al. 2013, AJ, 146, 81

Zhou, G., Bayliss, D., Hartman, J. D., et al. 2015, MNRAS, 451, 2263

Zucker, S. 2003, MNRAS, 342, 1291

Zucker, S., \& Mazeh, T. 1994, ApJ, 420, 806 